Journal für die reine und angewandte Mathematik (Crelle's Journal) 612 (2007), 17-33.

ISSN (Online) 1435-5345, ISSN (Print) 0075-4102, DOI: 10.1515/CRELLE.2007.082, November 2007.

\title{
THE GLOBAL QUANTUM DUALITY PRINCIPLE
}

\author{
FABIO GaVARini \\ Università degli Studi di Roma "Tor Vergata" — Dipartimento di Matematica \\ Via della Ricerca Scientifica 1, I-00133 Roma - ITALY
}

\begin{abstract}
Let $R$ be an integral domain, let $\hbar \in R \backslash\{0\}$ be such that $\mathbb{k}:=R / \hbar R$ is a field, and let $\mathcal{H A}$ be the category of torsionless (or flat) Hopf algebras over $R$. We call $H \in \mathcal{H} \mathcal{A}$ a "quantized function algebra" (=QFA), resp. "quantized restricted universal enveloping algebras" (=QrUEA), at $\hbar$

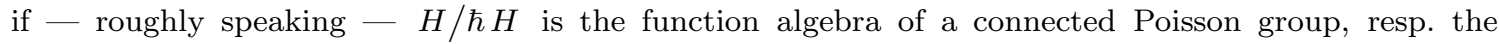
(restricted, if $R / \hbar R$ has positive characteristic) universal enveloping algebra of a (restricted) Lie bialgebra. Extending a result of Drinfeld, we establish an "inner" Galois' correspondence on $\mathcal{H} \mathcal{A}$, via two endofunctors, ()$^{\vee}$ and ()$^{\prime}$, of $\mathcal{H} \mathcal{A}$ such that $H^{\vee}$ is a QrUEA and $H^{\prime}$ is a QFA (for all $H \in \mathcal{H} \mathcal{A}$ ). In addition: (a) the image of ()$^{\vee}$, resp. of ()$^{\prime}$, is the full subcategory of all QrUEAs, resp. of all QFAs; (b) if $p:=\operatorname{Char}(\mathbb{k})=0$, the restrictions $\left.()^{\vee}\right|_{\text {QFAs }}$ and $\left.()^{\prime}\right|_{\text {QrUEAs }}$ yield equivalences inverse to each other; (c) if $p=0$, starting from a QFA over a Poisson group $G$, resp. from a QrUEA over a Lie bialgebra $\mathfrak{g}$, the functor ()$^{\vee}$, resp. ( $)^{\prime}$, gives a QrUEA, resp. a QFA, over the dual Lie bialgebra, resp. the dual Poisson group. Several, far-reaching applications are developed in detail in [Ga2-4].

$$
\begin{aligned}
& \text { "Dualitas dualitatum } \\
& \text { et omnia dualitas" }
\end{aligned}
$$$$
\text { N. Barbecue, "Scholia" }
$$

\section{Introduction}

Generalized "symmetries" in mathematics are described by Hopf algebras. Among these, the "geometrical" ones are of type $H=F[G]$, the algebra of regular functions over an algebraic group $G$, and $H=U(\mathfrak{g})(=\mathbf{u}(\mathfrak{g}))$, the (restricted, if the ground field $\mathbb{k}$ has positive characteristic) universal enveloping algebra of a (restricted) Lie algebra $\mathfrak{g}$. These notions of "geometrical symmetries" are generalized by quantum groups: roughly, these are Hopf algebras $H$ depending on a parameter $\hbar$ such that, setting $\hbar=0$, the Hopf algebra one gets is either of the type $F[G]$ hence $H$ is a quantized function algebra, in short QFA — or of the type $U(\mathfrak{g})$ or $\mathbf{u}(\mathfrak{g})$ (according to the characteristic of $\mathbb{k}$ ) - hence $H$ is a quantized restricted universal enveloping algebra, in short QrUEA. When a QFA exists whose specialization at $\hbar=0$ is $F[G]$, the algebraic group $G$ inherits a structure of Poisson (algebraic) group. Similarly, if a QrUEA exists whose specialization is $U(\mathfrak{g})$ or $\mathbf{u}(\mathfrak{g})$, the (restricted) Lie algebra $\mathfrak{g}$ inherits a structure of Lie bialgebra. Then, by general Poisson group theory, Poisson groups $G^{*}$ dual to $G$ and a Lie bialgebra $\mathfrak{g}^{*}$ dual to $\mathfrak{g}$ exist.

In this setting, three basic questions rise at once:

- (1) How can we produce quantum groups?

- (2) How can we characterize quantum groups (of either kind) among Hopf algebras?

- (3) What kind of relationship, if any, does exist between quantum groups over mutually dual Poisson groups, or mutually dual Lie bialgebras?

Keywords: Hopf algebras, Quantum Groups.

2000 Mathematics Subject Classification: Primary 16W30, 17B37, 20G42; Secondary 81R50.

Typeset by $\mathcal{A} \mathcal{M S}-\mathrm{TEX}_{\mathrm{E}}$ 
A first answer to (1) and (3) is given, for $\operatorname{Char}(\mathbb{k})=0$, by the "quantum duality principle", formulated by Drinfeld in terms of formal quantum groups (cf. [Dr], §7, and [Ga1]): it is a functorial recipe to get, out of a QFA over $G$, a QrUEA over $\mathfrak{g}^{*}$, and a QFA over $G^{*}$ out of a QrUEA over $\mathfrak{g}$.

In this paper I provide a global version of this principle, which answers questions (1) through (3). Indeed, I push Drinfeld's original method as far as possible, so to apply it to the category $\mathcal{H} \mathcal{A}$ of Hopf algebras which are torsion-free (or flat) over some integral domain, say $R$, and to do it for each $\hbar \in R \backslash\{0\}$ such that $\mathbb{k}:=R / \hbar R$ is a field. In fact, I extend Drinfeld's recipe so to define endofunctors of $\mathcal{H} \mathcal{A}$. The image of either functor is contained in a category of quantum groups (one gives QFAs, the other QrUEAs) so we answer question (1). If $\mathbb{k}$ has zero characteristic, when restricted to quantum groups these functors yield equivalences inverse to each other. Moreover, these equivalences exchange the types of quantum group (switching QFA with QrUEA) and the underlying Poisson symmetries (interchanging $G$ or $\mathfrak{g}$ with $G^{*}$ or $\mathfrak{g}^{*}$ ), thus solving (3). Other details show that these functors endow $\mathcal{H} \mathcal{A}$ with a (inner) Galois' correspondence, in which QFAs on one side and QrUEAs on the other side are the subcategories (in $\mathcal{H} \mathcal{A}$ ) of "fixed points" for the composition of both Drinfeld's functors (in suitable order): in particular, this answers question (2). Let me point out that, as my "Drinfeld's functors" are defined for each element $\hbar \in R$ as above, for any such $\hbar$ and for any $H$ in $\mathcal{H A}$ they yield two quantum groups, a QFA and a QrUEA, w.r.t. $\hbar$ itself. Thus we have a method to get, out of any single $H \in \mathcal{H} \mathcal{A}$, several quantum groups.

Further aspects, examples and applications of the main result are presented in [Ga2-4].

\section{ACKNOWLEDGEMENTS}

The author thanks P. Baumann, G. Carnovale, N. Ciccoli, A. D'Andrea, I. Damiani, B. Di Blasio, D. Fiorenza, L. Foissy, A. Frabetti, C. Gasbarri and E. Taft for many helpful discussions.

A special thank also to the referee for his valuable, fruitful comments and remarks.

\section{$\S 1$ Notation and terminology}

1.1 The classical setting. Let $\mathbb{k}$ be a fixed field of any characteristic. We call "algebraic group" the maximal spectrum $G$ associated to any commutative Hopf $\mathbb{k}$-algebra $H$; then $H$ is called the algebra of regular functions on $G$, denoted $F[G]$. We say that $G$ is connected if $F[G]$ has no non-trivial idempotents. We denote by $\mathfrak{m}_{e}$ the defining ideal of the unit element $e \in G$ (it is the augmentation ideal of $F[G]$ ); the cotangent space of $G$ at $e$ is $\mathfrak{g}^{\times}:=\mathfrak{m}_{e} / \mathfrak{m}_{e}^{2}$, which is naturally a Lie coalgebra. The tangent space of $G$ at $e$ is the dual space $\mathfrak{g}:=\left(\mathfrak{g}^{\times}\right)^{*}$ to $\mathfrak{g}^{\times}$, which is a Lie algebra. By $U(\mathfrak{g})$ we mean the universal enveloping algebra of $\mathfrak{g}$ : it is a connected cocommutative Hopf algebra, and there is a natural Hopf pairing (see $\S 1.2(a)$ ) between $F[G]$ and $U(\mathfrak{g})$. If $\operatorname{Char}(\mathbb{k})=p>0$, then $\mathfrak{g}$ is a restricted Lie algebra, and $\mathbf{u}(\mathfrak{g}):=U(\mathfrak{g}) /\left(\left\{x^{p}-x^{[p]}\right\}_{x \in \mathfrak{g}}\right)$ is the restricted universal enveloping algebra of $\mathfrak{g}$. To unify notation and terminology, when $\operatorname{Char}(\mathbb{k})=0$ we call any Lie algebra $\mathfrak{g}$ "restricted", by its "restricted universal enveloping algebra" we mean $U(\mathfrak{g})$, and we write $\mathcal{U}(\mathfrak{g}):=U(\mathfrak{g})$ if $\operatorname{Char}(\mathbb{k})=0$ and $\mathcal{U}(\mathfrak{g}):=\mathbf{u}(\mathfrak{g})$ if $\operatorname{Char}(\mathbb{k})>0$.

Let $H$ be a Hopf algebra over an integral domain $D$. We call $H$ a "function algebra" (FA in short) if it is commutative, with no non-trivial idempotents, and such that, if $p:=\operatorname{Char}(\mathbb{k})>0$, then $\eta^{p}=0$ for all $\eta$ in the kernel of the counit of $H$. If $D$ is a field, an FA is the algebra of regular functions of an algebraic group-scheme over $D$ which is connected and, if $\operatorname{Char}(\mathbb{k})>0$, is zero- 
dimensional of height 1 ; conversely, if $G$ is such a group-scheme then $F[G]$ has these properties. Instead, we call $H$ a "restricted universal enveloping algebra" (=rUEA) if it is cocommutative, connected, and generated by its primitive part. If $D$ is a field, an rUEA is the restricted universal enveloping algebra of some (restricted) Lie algebra over $D$; conversely, if $\mathfrak{g}$ is such a Lie algebra, then $\mathcal{U}(\mathfrak{g})$ has these properties (see, e.g., [Mo], Theorem 5.6.5, and references therein).

Now assume $G$ is a Poisson group (for this and other notions hereafter see [CP], but within an algebraic geometry setting). Then $F[G]$ is a Poisson Hopf algebra, whose Poisson bracket induces on $\mathfrak{g}^{\times}$a Lie bracket which makes it into a Lie bialgebra; hence $U\left(\mathfrak{g}^{\times}\right)$is a co-Poisson Hopf algebra too. Also, $\mathfrak{g}$ is a Lie bialgebra (in topological sense, if $G$ is infinite dimensional) too, and $U(\mathfrak{g}$ ) is a (maybe topological) co-Poisson Hopf algebra. The Hopf pairing between $F[G]$ and $U(\mathfrak{g})$ then is compatible with these additional co-Poisson and Poisson structures. Moreover, the perfect (=nondegenerate) evaluation pairing $\mathfrak{g} \times \mathfrak{g}^{\times} \rightarrow \mathbb{k}$ is compatible with the Lie bialgebra structure on either side (see $\S 1.2(b))$ : so $\mathfrak{g}$ and $\mathfrak{g}^{\times}$are Lie bialgebras dual to each other. In the sequel, we denote by $G^{\star}$ any connected algebraic Poisson group with $\mathfrak{g}$ as cotangent Lie bialgebra, and say it is dual to $G$.

If $H$ is a Hopf algebra we denote its Hopf operations by $\Delta$ (the coproduct), $\epsilon$ (the counit) and $S$ (the antipode), and we use standard $\sigma$-notation $\Delta(x)=\sum_{(x)} x_{(1)} \otimes x_{(2)}$ for all $x \in H$.

\section{Definition 1.2.}

(a) Let $H, K$ be Hopf algebras (maybe in topological sense) over a ring $R$. A Hopf (algebra) pairing between $H$ and $K$ is a pairing $\langle\rangle:, H \times K \longrightarrow R$ such that $\left\langle x, y_{1} y_{2}\right\rangle=\left\langle\Delta(x), y_{1} \otimes y_{2}\right\rangle:=$ $\sum_{(x)}\left\langle x_{(1)}, y_{1}\right\rangle\left\langle x_{(2)}, y_{2}\right\rangle,\left\langle x_{1} x_{2}, y\right\rangle=\left\langle x_{1} \otimes x_{2}, \Delta(y)\right\rangle:=\sum_{(y)}\left\langle x_{1}, y_{(1)}\right\rangle\left\langle x_{2}, y_{(2)}\right\rangle,\langle x, 1\rangle=\epsilon(x)$, $\langle 1, y\rangle=\epsilon(y),\langle S(x), y\rangle=\langle x, S(y)\rangle$, for all $x, x_{1}, x_{2} \in H, y, y_{1}, y_{2} \in K$.

(b) Let $\mathfrak{g}, \mathfrak{h}$ be Lie bialgebras (maybe in topological sense) over a ring $\mathbb{k}$. A Lie bialgebra pairing between $\mathfrak{g}$ and $\mathfrak{h}$ is a pairing $\langle\rangle:, \mathfrak{g} \times \mathfrak{h} \longrightarrow \mathbb{k}$ such that $\left\langle x,\left[y_{1}, y_{2}\right]\right\rangle=\left\langle\delta(x), y_{1} \otimes y_{2}\right\rangle:=$ $\sum_{[x]}\left\langle x_{[1]}, y_{1}\right\rangle\left\langle x_{[2]}, y_{2}\right\rangle,\left\langle\left[x_{1}, x_{2}\right], y\right\rangle=\left\langle x_{1} \otimes x_{2}, \delta(y)\right\rangle:=\sum_{[y]}\left\langle x_{1}, y_{[1]}\right\rangle\left\langle x_{2}, y_{[2]}\right\rangle$, for all $x, x_{1}, x_{2} \in$ $\mathfrak{g}$ and $y, y_{1}, y_{2} \in \mathfrak{h}$, with $\delta(x)=\sum_{[x]} x_{[1]} \otimes x_{[2]}$ and $\delta(y)=\sum_{[y]} y_{[1]} \otimes y_{[2]}$.

1.3 The quantum setting. Let $R$ be an integral domain, $F=F(R)$ its field of quotients. Let $\mathcal{M}$ be the category of torsion-free $R$-modules, $\mathcal{H} \mathcal{A}$ the category of all Hopf algebras in $\mathcal{M}$. Let $\mathcal{M}_{F}$ be the category of $F$-vector spaces, $\mathcal{H A}_{F}$ the category of all Hopf algebras in $\mathcal{M}_{F}$; for $M \in \mathcal{M}$, set $M_{F}:=F(R) \otimes_{R} M$. A subset $H \subseteq \mathbb{H} \in \mathcal{H} \mathcal{A}_{F}$ is called an $R$-integer form (or an $R$-form) of $\mathbb{H}$ iff $H$ is a Hopf $R$-subalgebra of $\mathbb{H}$ (hence in particular $H \in \mathcal{H} \mathcal{A}$ ) and $H_{F}:=F(R) \otimes_{R} H=\mathbb{H}$.

Let $\hbar \in R \backslash\{0\}$ be prime (fixed throughout), and $\mathbb{k}:=R /(\hbar)=R / \hbar R$. For any $R$-module $M$, set $\left.M_{\hbar}\right|_{\hbar=0}:=M / \hbar M=\mathbb{k} \otimes_{R} M$ (the specialization of $M$ at $\left.\hbar=0\right)$ and $M_{\infty}:=\bigcap_{n=0}^{+\infty} \hbar^{n} M$. Finally, for any $H \in \mathcal{H} \mathcal{A}$, let $I_{H}:=\operatorname{Ker}(H \stackrel{\epsilon}{\longrightarrow} R \stackrel{\hbar \mapsto 0}{\longrightarrow} \mathbb{k})$, and set $I_{H}^{\infty}:=\bigcap_{n=0}^{+\infty} I_{H}^{n}$.

Definition 1.4. ("Global quantum groups") Let $\hbar \in R \backslash\{0\}$ be a prime, and $\mathbb{k}:=R / \hbar R$.

(a) We call quantized restricted universal enveloping algebra (at $\hbar)$ - in short, QrUEA — any $\mathcal{U}_{\hbar} \in \mathcal{H} \mathcal{A}$ such that $\left.\mathcal{U}_{\hbar}\right|_{\hbar=0}:=\mathcal{U}_{\hbar} / \hbar \mathcal{U}_{\hbar}$ is a restricted universal enveloping algebra (an rUEA) over $\mathbb{k}$.

$W$ e call $\mathcal{Q} r \mathcal{U E \mathcal { A }}$ the full subcategory of $\mathcal{H} \mathcal{A}$ whose objects are all the QrUEAs (at $\hbar)$.

(b) We call quantized function algebra (at $\hbar)$ - in short, QFA - any $F_{\hbar} \in \mathcal{H} \mathcal{A}$ such that $\left.F_{\hbar}\right|_{\hbar=0}:=F_{\hbar} / \hbar F_{\hbar}$ is a function algebra (an $F A$ ) over $\mathbb{k}$, and $\left(F_{\hbar}\right)_{\infty}=I_{F_{\hbar}}^{\infty}$ (notation of $\S 1.3$ ).

$W$ e call $\mathcal{Q F A}$ the full subcategory of $\mathcal{H} \mathcal{A}$ whose objects are all the QFAs (at $\hbar$ ). 
Remark 1.5: If $\mathcal{U}_{\hbar}$ is a QrUEA (at $\hbar$ ) then $\left.\mathcal{U}_{\hbar}\right|_{\hbar=0}$ is a co-Poisson Hopf algebra, w.r.t. the Poisson cobracket $\delta$ defined as follows: if $\left.x \in \mathcal{U}_{\hbar}\right|_{\hbar=0}$ and $x^{\prime} \in \mathcal{U}_{\hbar}$ gives $x=x^{\prime} \bmod \hbar \mathcal{U}_{\hbar}$, then $\delta(x):=\left(\hbar^{-1}\left(\Delta\left(x^{\prime}\right)-\Delta^{\mathrm{op}}\left(x^{\prime}\right)\right)\right) \bmod \hbar\left(\mathcal{U}_{\hbar} \otimes \mathcal{U}_{\hbar}\right)$. So, if $\mathbb{k}$ is a field, then $\left.\mathcal{U}_{\hbar}\right|_{\hbar=0} \cong \mathcal{U}(\mathfrak{g})$ for some Lie algebra $\mathfrak{g}$, and by $[\mathrm{Dr}], \S 3$, the restriction of $\delta$ makes $\mathfrak{g}$ into a Lie bialgebra; then I shall write $\mathcal{U}_{\hbar}=\mathcal{U}_{\hbar}(\mathfrak{g})$. Similarly, if $F_{\hbar}$ is a QFA at $\hbar$, then $\left.F_{\hbar}\right|_{\hbar=0}$ is a Poisson Hopf algebra, w.r.t. the Poisson bracket $\{$,$\} defined as follows: if x,\left.y \in F_{\hbar}\right|_{\hbar=0}$ and $x^{\prime}, y^{\prime} \in F_{\hbar}$ give $x=x^{\prime} \bmod \hbar F_{\hbar}, y=y^{\prime}$ $\bmod \hbar F_{\hbar}$, then $\{x, y\}:=\left(\hbar^{-1}\left(x^{\prime} y^{\prime}-y^{\prime} x^{\prime}\right)\right) \bmod \hbar F_{\hbar}$. Thus, if $\mathbb{k}$ is a field, $\left.F_{\hbar}\right|_{\hbar=0} \cong F[G]$ for some connected Poisson algebraic group $G$ : in this case I shall write $F_{\hbar}=F_{\hbar}[G]$.

\section{Definition 1.6.}

(a) Let $R$ be an integral domain, and let $F$ be its field of fractions. Given two $F$-modules $\mathbb{A}$, $\mathbb{B}$, and an $F$-bilinear pairing $\mathbb{A} \times \mathbb{B} \longrightarrow F$, for any $R$-submodule $A \subseteq \mathbb{A}$ and $B \subseteq \mathbb{B}$ we define $A^{\bullet}:=\{b \in \mathbb{B} \mid\langle A, b\rangle \subseteq R\}$ and $B^{\bullet}:=\{a \in \mathbb{A} \mid\langle a, B\rangle \subseteq R\}$.

(b) Let $R$ be an integral domain. Given $H, K \in \mathcal{H} \mathcal{A}$, we say that $H$ and $K$ are dual to each other if there exists a perfect Hopf pairing between them for which $H=K^{\bullet}$ and $K=H^{\bullet}$.

\section{$\S 2$ The global quantum duality principle}

2.1 Drinfeld's functors. (Cf. [Dr], $\S 7$ ) Let $R, F, \mathcal{H A}$ and $\hbar \in R \backslash\{0\}$ be as in $\S 1.3$. For any $H \in \mathcal{H} \mathcal{A}$, let $I=I_{H}:=\operatorname{Ker}(H \stackrel{\epsilon}{\longrightarrow} R \stackrel{\hbar \mapsto 0}{\longrightarrow} R / \hbar R=\mathbb{k})=\operatorname{Ker}(H \stackrel{\hbar \mapsto 0}{\longrightarrow} H / \hbar H \stackrel{\bar{\epsilon}}{\longrightarrow} \mathbb{k})$, as in $\S 1.3$, where $\bar{\epsilon}$ denotes the counit of $\left.H\right|_{\hbar=0}$. I define

$$
H^{\vee}:=\sum_{n \geq 0} \hbar^{-n} I^{n}=\sum_{n \geq 0}\left(\hbar^{-1} I\right)^{n}=\bigcup_{n \geq 0}\left(\hbar^{-1} I\right)^{n} \quad\left(\subseteq H_{F}\right) .
$$

If $J=J_{H}:=\operatorname{Ker}\left(\epsilon_{H}\right)$ then $I=J+\hbar R \cdot 1_{H}$, so $H^{\vee}=\sum_{n \geq 0} \hbar^{-n} J^{n}=\sum_{n \geq 0}\left(\hbar^{-1} J\right)^{n}$ too.

Given any Hopf algebra $H$, for every $n \in \mathbb{N}$ define $\Delta^{n}: H \longrightarrow H^{\otimes n}$ by $\Delta^{0}:=\epsilon, \Delta^{1}:=\operatorname{id}_{H}$, and $\Delta^{n}:=\left(\Delta \otimes \mathrm{id}_{H}^{\otimes(n-2)}\right) \circ \Delta^{n-1}$ if $n>2$. For any ordered subset $\Sigma=\left\{i_{1}, \ldots, i_{k}\right\} \subseteq\{1, \ldots, n\}$ with $i_{1}<\cdots<i_{k}$, define the morphism $j_{\Sigma}: H^{\otimes k} \longrightarrow H^{\otimes n}$ by $j_{\Sigma}\left(a_{1} \otimes \cdots \otimes a_{k}\right):=b_{1} \otimes \cdots \otimes b_{n}$ with $b_{i}:=1$ if $i \notin \Sigma$ and $b_{i_{m}}:=a_{m}$ for $1 \leq m \leq k$. Set $\Delta_{\Sigma}:=j_{\Sigma} \circ \Delta^{k}, \Delta_{\emptyset}:=\Delta^{0}$, and $\delta_{\Sigma}:=\left.\sum_{\Sigma^{\prime} \subset \Sigma}(-1)^{n-\mid \Sigma^{\prime}}\right|_{\Sigma_{\Sigma^{\prime}}}, \delta_{\emptyset}:=\epsilon$; this admits the inverse formula $\Delta_{\Sigma}=\sum_{\Psi \subseteq \Sigma} \delta_{\Psi}$. We shall use notation $\delta_{0}:=\delta_{\emptyset}, \delta_{n}:=\delta_{\{1,2, \ldots, n\}}$, and the useful formula $\delta_{n}=\left(\operatorname{id}_{H}-\epsilon\right)^{\otimes n} \circ \Delta^{n}\left(n \in \mathbb{N}_{+}\right)$.

Now consider again any $H \in \mathcal{H} \mathcal{A}$ and $\hbar \in R \backslash\{0\}$ as in $\S 1.3$. Then I define

$$
H^{\prime}:=\left\{a \in H \mid \delta_{n}(a) \in \hbar^{n} H^{\otimes n}, \forall n \in \mathbb{N}\right\} \quad(\subseteq H) .
$$

Now I can state the main result of the paper:

Theorem 2.2. ("The Global Quantum Duality Principle") Assume that $\mathbb{k}:=R / \hbar R$ is a field.

(a) The assignment $H \mapsto H^{\vee}$, resp. $H \mapsto H^{\prime}$, defines a functor ()$^{\vee}: \mathcal{H} \mathcal{A} \longrightarrow \mathcal{H} \mathcal{A}$, resp. ()$^{\prime}: \mathcal{H} \mathcal{A} \longrightarrow \mathcal{H} \mathcal{A}$, whose image lies in $\mathcal{Q} r \mathcal{U E} \mathcal{A}$, resp. in $\mathcal{Q F} \mathcal{A}$. Moreover, for all $H \in \mathcal{H} \mathcal{A}$ we have $H \subseteq\left(H^{\vee}\right)^{\prime}$ and $H \supseteq\left(H^{\prime}\right)^{\vee}$, hence $H^{\vee}=\left(\left(H^{\vee}\right)^{\prime}\right)^{\vee}$ and $H^{\prime}=\left(\left(H^{\prime}\right)^{\vee}\right)^{\prime}$. In addition, if $H \in \mathcal{H} \mathcal{A}$ is flat, then $H^{\vee}$ and $H^{\prime}$ are flat as well.

(b) Assume that $\operatorname{Char}(\mathbb{k})=0$. Then for any $H \in \mathcal{H} \mathcal{A}$ we have

$$
H=\left(H^{\vee}\right)^{\prime} \Longleftrightarrow H \in \mathcal{Q} \mathcal{F A} \quad \text { and } \quad H=\left(H^{\prime}\right)^{\vee} \Longleftrightarrow H \in \mathcal{Q} r \mathcal{U E} \mathcal{A}
$$

thus ( $)^{\vee}$ and ( )' restrict to equivalences, inverse to each other, between $\mathcal{Q F \mathcal { A }}$ and $\mathcal{Q} r \mathcal{U E \mathcal { A }}$. 
(c) ("Quantum Duality Principle") Assume that Char $(\mathbb{k})=0$. Then

$$
\left.F_{\hbar}[G]^{\vee}\right|_{\hbar=0}:=F_{\hbar}[G]^{\vee} / \hbar F_{\hbar}[G]^{\vee}=U\left(\mathfrak{g}^{\times}\right),\left.\quad U_{\hbar}(\mathfrak{g})^{\prime}\right|_{\hbar=0}:=U_{\hbar}(\mathfrak{g})^{\prime} / \hbar U_{\hbar}(\mathfrak{g})^{\prime}=F\left[G^{\star}\right]
$$

(cf. $\S \S 1.1,1.5)$ where the choice of $G^{\star}$ (among all the connected Poisson algebraic groups with tangent Lie bialgebra $\left.\mathfrak{g}^{\star}\right)$ depends on the choice of $U_{\hbar}(\mathfrak{g})$. In other words, $F_{\hbar}[G]^{\vee}$ is a $\operatorname{QrUEA}$ for the Lie bialgebra $\mathfrak{g}^{\times}$, and $U_{\hbar}(\mathfrak{g})^{\prime}$ is a QFA for the Poisson group $G^{\star}$.

(d) Assume that $\operatorname{Char}(\mathbb{k})=0$. Let $F_{\hbar} \in \mathcal{Q} \mathcal{F} \mathcal{A}, U_{\hbar} \in \mathcal{Q} r \mathcal{U} \mathcal{E} \mathcal{A}$ be dual to each other (w.r.t. some pairing). Then $F_{\hbar}^{\vee}$ and $U_{\hbar}^{\prime}$ are dual to each other (w.r.t. the same pairing).

(e) Assume that Char $(\mathbb{k})=0$. Then for any $\mathbb{H} \in \mathcal{H} \mathcal{A}_{F}$ the following are equivalent:

$(e-1) \mathbb{H}$ has an $R$-integer form $H_{(f)}$ which is a $Q F A$ at $\hbar$;

$(e-2) \mathbb{H}$ has an $R$-integer form $H_{(u)}$ which is a QrUEA at $\hbar$.

Remarks 2.3: (a) In [Ga2] the effect of Drinfeld's functors on some popular quantum groups or other Hopf algebras is studied in detail. An important application to "classical" Hopf algebras is explained in [Ga3]: a special case of it, regarding the Nottingham group, is studied in [Ga4].

(b) Theorem 2.2 can be still partially generalized, see $\S 4.10$ at the end of the paper.

\section{$\S 3$ General properties of Drinfeld's functors}

We begin with a few technicalities, then pass to the first relevant results. Fix $R$ and $\hbar$ as in $\S 1.3$.

Lemma 3.1. Let $H \in \mathcal{H} \mathcal{A}$, and set $\bar{H}:=H / H_{\infty}$ (notation of $\S 1.3$ ). Then:

(a) $H_{\infty}=\left(H^{\prime}\right)_{\infty}, H_{\infty} \subseteq\left(H^{\vee}\right)_{\infty}, H_{\infty}$ is a Hopf ideal and subcoalgebra of $H$, and $(\bar{H})_{\infty}=\{0\}$. Moreover, there are natural isomorphisms $(\bar{H})^{\vee} \cong H^{\vee} / H_{\infty}$ and $(\bar{H})^{\prime} \cong H^{\prime} / H_{\infty}$.

(b) $\bar{H} \in \mathcal{H} \mathcal{A}$, and $\left.\bar{H}\right|_{\hbar=0}=\left.H\right|_{\hbar=0}$. In particular, if $H$ is a $Q F A$, then $\bar{H}$ is a $Q F A$ too, and if $H$ is a $\operatorname{QrUEA}$, then $\bar{H}$ is a $\operatorname{QrUEA}$ too.

Lemma 3.2. ([KT], Lemma 3.2) Let $H \in \mathcal{H} \mathcal{A}, a, b \in H$, and $\Phi \subseteq \mathbb{N}$ a finite subset. Then

(a) $\delta_{\Phi}(a b)=\sum_{\Lambda \cup Y=\Phi} \delta_{\Lambda}(a) \delta_{Y}(b) ;$

(b) if $\Phi \neq \emptyset$, then $\quad \delta_{\Phi}(a b-b a)=\sum_{\substack{\Lambda \cup Y=\Phi \\ \Lambda \cap Y \neq \emptyset}}\left(\delta_{\Lambda}(a) \delta_{Y}(b)-\delta_{Y}(b) \delta_{\Lambda}(a)\right)$

From now on, we make the following assumption: $\mathbb{k}:=R / \hbar R$ is a field.

Proposition 3.3. $H \mapsto H^{\vee}$ and $H \mapsto H^{\prime}$ gives well-defined endofunctors of $\mathcal{H} \mathcal{A}$, which preserve flatness. They enjoy $H \subseteq\left(H^{\vee}\right)^{\prime}, H \supseteq\left(H^{\prime}\right)^{\vee}$, so $H^{\vee}=\left(\left(H^{\vee}\right)^{\prime}\right)^{\vee}, H^{\prime}=\left(\left(H^{\prime}\right)^{\vee}\right)^{\prime}$, for all $H \in \mathcal{H} \mathcal{A}$.

Proof. Given $H \in \mathcal{H} \mathcal{A}$, clearly $H^{\vee}$ and $H^{\prime}$ are torsion-free, hence $H^{\vee}, H^{\prime} \in \mathcal{M}$. Moreover, flatness is preserved when taking submodules and/or localizing, so $H^{\vee}$ and $H^{\prime}$ are flat is $H$ is flat.

Since $J:=\operatorname{Ker}\left(\epsilon_{H}\right)$ is a Hopf ideal of $H$, we see at once that $H^{\vee} \in \mathcal{H} \mathcal{A}$ also.

On the other hand, $H^{\prime}$ is a unital $R$-subalgebra of $H$ thanks to Lemma 3.2(a) and the very definitions. Moreover, $\Delta^{n} \circ S=S^{\otimes n} \circ \Delta^{n}$ implies $\delta_{n} \circ S=S^{\otimes n} \circ \delta_{n}(n \in \mathbb{N})$, which yields $S\left(H^{\prime}\right)=H^{\prime}$. Thus we still need only to prove that $H^{\prime}$ is a subcoalgebra, namely $\Delta\left(H^{\prime}\right) \subseteq H^{\prime} \otimes H^{\prime}$. 
Consider $D_{H}^{\prime}:=\left\{z \in H \otimes H \mid\left(\delta_{r} \otimes \delta_{s}\right)(z) \in \hbar^{r+s} H^{\otimes r} \otimes H^{\otimes s}, \forall r, s \in \mathbb{N}\right\}$. The coassociativity of $\Delta$ yields $\left(\delta_{r} \otimes \delta_{s}\right) \circ \Delta=\delta_{r+s}$, whence we get $\Delta\left(H^{\prime}\right) \subseteq D_{H}^{\prime}$. Therefore, the claim will follow once we prove the identity $D_{H}^{\prime}=H^{\prime} \otimes H^{\prime}$. The key point is to prove that $D_{H}^{\prime} \subseteq H^{\prime} \otimes H^{\prime}$, the converse inclusion being trivial; to this end, we shall resort to completions. Note that, by Lemma 3.1, we can reduce to prove the main statement for $\bar{H}^{\prime}$. Even more, one clearly has $D_{H}^{\prime}=H^{\prime} \otimes H^{\prime}$ if and only if $D \frac{\prime}{H}=\bar{H}^{\prime} \otimes \bar{H}^{\prime}$. Therefore, we can assume that $H_{\infty}=\{0\}$.

Let $\widehat{R}$ and $\widehat{H}$ respectively be the $\hbar$-adic completion of $R$ and $H$ : then $\widehat{H}$ is a separated complete topological $\widehat{R}$-module, hence it is topologically free, and is a topological Hopf algebra. Its coproduct takes values into the $\hbar$-adic completion $H \widehat{\otimes} H$ of $H \otimes H$, and $H \widehat{\otimes} H=\widehat{H} \widehat{\otimes} \widehat{H}=$ $\widehat{H \otimes H}$. As $H$ embeds into $\widehat{H}$ (because $H_{\infty}=\{0\}$ ), we identify $H$ itself with a (dense) Hopf $R$-subalgebra of $\widehat{H}$. Then $\hbar^{n} H=H \bigcap\left(\hbar^{n} \widehat{H}\right)$ and $\widehat{H} / \hbar^{n} \widehat{H}=H / \hbar^{n} H$, for all $n \in \mathbb{N}$.

Let $\widehat{H}^{\prime}:=\left\{a \in \widehat{H} \mid \delta_{n}(a) \in \hbar^{n} \widehat{H}^{\widehat{\otimes}}, \forall n \in \mathbb{N}\right\}$. Then clearly $H^{\prime}=\widehat{H}^{\prime} \cap H$. Similarly, define $(\widehat{H \otimes H})^{\prime}$ simply taking $H \otimes H$ instead of $H$, and define $D_{\widehat{H}}^{\prime}$ just mimicking the definition of $D_{H}^{\prime}$.

Now, Proposition 2.1 in $[\mathrm{EH}]$ proves that $D_{\widehat{H}}^{\prime}=(\widehat{H \otimes H})^{\prime}$; to be precise, the statement in $[\mathrm{EH}]$ is for quantized universal enveloping algebras, but the arguments used therein can be easily modified as to apply to our $\widehat{H}$ as well. In addition, one has also $(\widehat{H \otimes H})^{\prime}=\widehat{H}^{\prime} \bar{\otimes} \widehat{H}^{\prime}$, where $\bar{\otimes}$ denotes a topological tensor product (w.r.t. the weak topology, see [EH] or [Ga1] for details), hence one gets $(\widehat{H \otimes H})^{\prime} \cap(H \otimes H)=\left(\widehat{H}^{\prime} \bar{\otimes} \widehat{H}^{\prime}\right) \cap(H \otimes H)=\left(\widehat{H}^{\prime} \cap H\right) \otimes\left(\widehat{H}^{\prime} \cap H\right)=H^{\prime} \otimes H^{\prime}$. But then, noting that $D_{H}^{\prime}=D_{\widehat{H}}^{\prime} \cap(H \otimes H)$, the identity $D_{\widehat{H}}^{\prime}=(\widehat{H \otimes H})^{\prime}$ eventually yields $D_{H}^{\prime}=D_{\widehat{H}}^{\prime} \cap(H \otimes H)=(\widehat{H \otimes H})^{\prime} \cap(H \otimes H)=H^{\prime} \otimes H^{\prime}$, q.e.d.

The outcome is that $H^{\prime} \in \mathcal{H} \mathcal{A}$, hence ()$^{\prime}$ is well defined on objects and takes values into $\mathcal{H} \mathcal{A}$.

As to morphisms, let $\varphi \in \operatorname{Mor}_{\mathcal{H A}}(H, K)$. Then its scalar extension $\varphi_{F(R)}$ defines $\varphi^{\vee}:=$ $\left.\varphi_{F(R)}\right|_{H^{\vee}} \in \operatorname{Mor}_{\mathcal{H A}}\left(H^{\vee}, K^{\vee}\right)$; similarly one defines $\varphi^{\prime}:=\left.\varphi\right|_{H^{\prime}} \in \operatorname{Mor}_{\mathcal{H A}}\left(H^{\prime}, K^{\prime}\right)$ as well.

Finally, let $H \in \mathcal{H} \mathcal{A}$. For $n \in \mathbb{N}$ we have $\delta_{n}(H) \subseteq J_{H}^{\otimes n}$ (see $\S 2.1$ ), thus $\delta_{n}(H) \subseteq J_{H}^{\otimes n}=$ $\hbar^{n}\left(\hbar^{-1} J_{H}\right)^{\otimes n} \subseteq \hbar^{n}\left(H^{\vee}\right)^{\otimes n}$, which gives $H \subseteq\left(H^{\vee}\right)^{\prime}$. For the rest, let $I^{\prime}:=\operatorname{Ker}\left(H^{\prime} \stackrel{\epsilon}{\longrightarrow} R \stackrel{\hbar \mapsto 0}{\longrightarrow} \mathbb{k}\right)$; as $\left(H^{\prime}\right)^{\vee}:=\bigcup_{n=0}^{\infty}\left(\hbar^{-1} I^{\prime}\right)^{n}$, to show that $H \supseteq\left(H^{\prime}\right)^{\vee}$ it is enough to prove that $H \supseteq \hbar^{-1} I^{\prime}$. So let $x^{\prime} \in I^{\prime}$ : then $\delta_{1}\left(x^{\prime}\right) \in \hbar H$, hence $x^{\prime}=\delta_{1}\left(x^{\prime}\right)+\epsilon\left(x^{\prime}\right) \in \hbar H$, thus $\hbar^{-1} x^{\prime} \in H$.

In the end, the last two identities follow directly from the inclusions that we just proved.

Theorem 3.4. Let $H \in \mathcal{H} \mathcal{A}$. Then $H^{\vee} \in \mathcal{Q} r \mathcal{U E} \mathcal{A}$.

Proof. By definition $H^{\vee}$ is generated by $J^{\vee}:=\hbar^{-1} J$, hence $\left.H^{\vee}\right|_{\hbar=0}$ is generated by $\left.J^{\vee}\right|_{\hbar=0}$. Now pick $j^{\vee} \in J^{\vee}$ and $j:=\hbar j^{\vee} \in J$; then $\Delta(j)=\delta_{2}(j)+j \otimes 1+1 \otimes j-\epsilon(j) \cdot 1 \otimes 1 \in j \otimes 1+1 \otimes j+J \otimes J$, therefore $\Delta\left(j^{\vee}\right) \in j^{\vee} \otimes 1+1 \otimes j^{\vee}+\hbar^{+1} J^{\vee} \otimes J^{\vee}$, whence $\Delta\left(\overline{j^{\vee}}\right)=\overline{j^{\vee}} \otimes 1+1 \otimes \overline{j^{\vee}}$ for $\overline{j^{\vee}}:=j^{\vee}$ $\left.\bmod \hbar H^{\vee} \in H^{\vee}\right|_{\hbar=0}$. Thus $\left.H^{\vee}\right|_{\hbar=0}$ is generated by its primitive part $P\left(\left.H^{\vee}\right|_{\hbar=0}\right)$, hence it is cocommutative. Even more, this fact enables us to apply Lemma 5.5.1 in [Mo] to $\left.H^{\vee}\right|_{\hbar=0}$, which then proves that $\left.H^{\vee}\right|_{\hbar=0}$ is connected (as a Hopf algebra). Thus $\left.H^{\vee}\right|_{\hbar=0}$ is cocommutative, connected and generated by its primitive part: that is, it is an rUEA, so that $H^{\vee} \in \mathcal{Q} r \mathcal{U E} \mathcal{A}$.

Theorem 3.5. Let $H \in \mathcal{H} \mathcal{A}$. Then $H^{\prime} \in \mathcal{Q \mathcal { F }}$.

Proof. First, $\left.H^{\prime}\right|_{\hbar=0}$ is commutative as a consequence of Lemma 3.2(b) (cf. [KT], Proposition 3.5). Second, we show that $\left(H^{\prime}\right)_{\infty}=\left(I^{\prime}\right)^{\infty}$. For later use, set $I:=I_{H}, J:=J_{H}, J^{\prime}:=J_{H^{\prime}}, I^{\prime}:=I_{H^{\prime}}$. 
By definition $\hbar H^{\prime} \subseteq I^{\prime}$, whence $H_{\infty}^{\prime}:=\bigcap_{n=0}^{+\infty} \hbar^{n} H^{\prime} \subseteq \bigcap_{n=0}^{+\infty}\left(I^{\prime}\right)^{n}=:\left(I^{\prime}\right)^{\infty}$, i.e. $\left(H^{\prime}\right)_{\infty} \subseteq\left(I^{\prime}\right)^{\infty}$. Conversely, $I^{\prime}=\hbar H^{\prime}+J^{\prime}$ with $\hbar H^{\prime} \subseteq \hbar H$ and $J^{\prime}=\delta_{1}\left(J^{\prime}\right) \subseteq \hbar H$ : thus $I^{\prime} \subseteq \hbar H$, hence $\left(I^{\prime}\right)^{\infty} \subseteq \bigcap_{n=0}^{+\infty} \hbar^{n} H=: H_{\infty}$. Now definitions give $H_{\infty} \subseteq H^{\prime}$ and $\hbar^{\ell} H_{\infty}=H_{\infty}$ for all $\ell \in \mathbb{Z}$, so $\hbar^{-n}\left(I^{\prime}\right)^{\infty} \subseteq \hbar^{-n} H_{\infty}=H_{\infty} \subseteq H^{\prime}$ hence $\left(I^{\prime}\right)^{\infty} \subseteq \hbar^{n} H^{\prime}$ for all $n \in \mathbb{N}$, thus finally $\left(I^{\prime}\right)^{\infty} \subseteq\left(H^{\prime}\right)_{\infty}$.

Third, we prove that $\left.H^{\prime}\right|_{\hbar=0}$ has no non-trivial idempotents. Let $a \in H^{\prime}$ be such that $\bar{a}:=a$ $\left.\bmod \hbar H^{\prime} \in H^{\prime}\right|_{\hbar=0}$ is idempotent, i.e. $\bar{a}^{2}=\bar{a}$ : then $a^{2}=a+\hbar c$ for some $c \in H^{\prime}$. Set $a_{0}:=\epsilon(a)$, $a_{1}:=\delta_{1}(a)$, and $c_{0}:=\epsilon(c), c_{1}:=\delta_{1}(c) ;$ as $a, c \in H^{\prime}$ we have $a_{1}, c_{1} \in \hbar H \cap J=\hbar J$. Applying $\delta_{n}$ to $a^{2}=a+\hbar c$ we get $\sum_{\Lambda \cup Y=\{1, \ldots, n\}} \delta_{\Lambda}(a) \delta_{Y}(a)=\delta_{n}\left(a^{2}\right)=\delta_{n}(a)+\hbar \delta_{n}(c)$ (for all $n \in \mathbb{N}_{+}$), thanks to Lemma 3.2(a). Since $a, c \in H^{\prime}$ we have $\delta_{n}(a), \delta_{n}(c) \in \hbar^{n} H^{\otimes n}$ (for all $n$ ), hence

$$
\delta_{n}(a) \equiv \sum_{\Lambda \cup Y=\{1, \ldots, n\}} \delta_{\Lambda}(a) \delta_{Y}(a)=2 \delta_{0}(a) \delta_{n}(a)+\sum_{\substack{\Lambda \cup Y=\{1, \ldots, n\} \\ \Lambda, Y \neq \emptyset}} \delta_{\Lambda}(a) \delta_{Y}(a) \quad \bmod \hbar^{n+1} H^{\otimes n}
$$

for all $n \in \mathbb{N}_{+}$, which, recalling that $a_{0}:=\delta_{0}(a)$, gives (for all $n \in \mathbb{N}_{+}$)

$$
\left(1-2 a_{0}\right) \delta_{n}(a) \equiv \sum_{\substack{\Lambda, Y \neq \emptyset \\ \Lambda \cup Y=\{1, \ldots, n\}}} \delta_{\Lambda}(a) \delta_{Y}(a) \quad \bmod \hbar^{n+1} H^{\otimes n}
$$

Now, applying $\epsilon$ to $a^{2}=a+\hbar c$ gives $a_{0}^{2}=a_{0}+\hbar c_{0}$. This implies $\left(1-2 a_{0}\right) \notin \hbar H$. In fact, if $\left(1-2 a_{0}\right)=\hbar \alpha(\alpha \in \hbar H)$, then $\hbar^{2} \alpha^{2}=\left(1-2 a_{0}\right)^{2}=1-4 a_{0}+4 a_{0}^{2}=1+4 \hbar c_{0}$, which entails $1 \in \hbar R$, a contradiction. From $\left(1-2 a_{0}\right) \notin \hbar H$ and (3.1), for all $n \in \mathbb{N}_{+}$, an easy induction gives $\delta_{n}(a) \in \hbar^{n+1} H^{\otimes n}$ for all $n$. Now take $a_{1}=a-a_{0}=\hbar \alpha$ for some $\alpha \in \hbar J$ : then $\delta_{0}(\alpha)=\epsilon(\alpha)=0$ and $\delta_{n}(\alpha)=\hbar^{-1} \delta_{n}(a) \in \hbar^{n} H^{\otimes n}$ (for all $n$ ), hence $\alpha \in H^{\prime}$. Thus $a=a_{0}+\hbar \alpha \equiv a_{0} \bmod \hbar H^{\prime}$, whence $\bar{a}=\left.\overline{a_{0}} \in H^{\prime}\right|_{\hbar=0}$; then ${\overline{a_{0}}}^{2}=\overline{a_{0}} \in \mathbb{k}$ gives us $\overline{a_{0}} \in\{0,1\}$, hence $\bar{a}=\overline{a_{0}} \in\{0,1\}$, q.e.d.

Finally, assume that $p:=\operatorname{Char}(\mathbb{k})>0$; then we have to show that $\bar{\eta}^{p}=0$ for each $\bar{\eta}^{p} \in$ $J_{\left.H^{\prime}\right|_{\hbar=0}}$, or simply $\eta^{p} \in \hbar J_{H^{\prime}}$ for each $\eta \in J_{H^{\prime}}$. Indeed, for any $n \in \mathbb{N}$ by the multiplicativity of $\Delta^{n}$ and $\Delta^{n}(\eta)=\sum_{\Lambda \subseteq\{1, \ldots, n\}} \delta_{\Lambda}(\eta)$ (cf. $\left.\S 2.1\right)$ we get that $\Delta^{n}\left(\eta^{p}\right)=\left(\sum_{\Lambda \subseteq\{1, \ldots, n\}} \delta_{\Lambda}(\eta)\right)^{p}$ belongs to $\sum_{\Lambda \subseteq\{1, \ldots, n\}} \delta_{\Lambda}(\eta)^{p}+\sum_{\substack{e_{1}, \ldots, e_{p}<p \\ e_{1}+\cdots, e_{p}=p}}\left(\begin{array}{c}p \\ e_{1}, \ldots, e_{p}\end{array}\right) \sum_{\Lambda_{k} \subseteq\{1, \ldots, n\}, \forall k} \prod_{k=1}^{p} \delta_{\Lambda_{k}}(\eta)^{e_{k}}+\hbar \sum_{k=0}^{n-1} \sum_{\substack{\Psi \subseteq\{1, \ldots, n\} \\|\Psi|=k}} j_{\Psi}\left(J_{H^{\prime}}^{\otimes k}\right)+\hbar J_{H^{\prime}}^{\otimes n}$ because $\delta_{\Lambda}(\eta) \in j_{\Lambda}\left(J_{H^{\prime}}^{\otimes|\Lambda|}\right)$ (for all $\left.\Lambda \subseteq\{1, \ldots, n\}\right)$ and $\left[J_{H^{\prime}}, J_{H^{\prime}}\right] \subseteq \hbar J_{H^{\prime}}$. Then

$$
\delta^{n}\left(\eta^{p}\right)=\left(\mathrm{id}_{H}-\epsilon\right)^{\otimes n}\left(\Delta^{n}\left(\eta^{p}\right)\right) \in \delta_{n}(\eta)^{p}+\sum_{\substack{e_{1}, \ldots, e_{p}<p \\
e_{1}+\cdots e_{p}=p}}^{\left(\begin{array}{c}
p \\
e_{1}, \ldots, e_{p}
\end{array}\right)} \sum_{\cup_{k} \Lambda_{k}=\{1, \ldots, n\}} \prod_{k=1}^{p} \delta_{\Lambda_{k}}(\eta)^{e_{k}}+\hbar J_{H^{\prime}}^{\otimes n} .
$$

Now, $\delta^{n}(\eta)^{p} \in\left(\hbar^{n} H^{\otimes n}\right)^{p} \subseteq \hbar^{n+1} H^{\otimes n}$ since $\eta \in H^{\prime}$; similarly $\prod_{k=1}^{p} \delta_{\Lambda_{k}}(\eta)^{e_{k}} \in \hbar^{\sum_{k}\left|\Lambda_{k}\right| e_{k}} H^{\otimes n} \subseteq$ $\hbar^{n} H^{\otimes n}$ if $\bigcup_{k=1}^{n} \Lambda_{k}=\{1, \ldots, n\}$. Moreover, $\left(\begin{array}{c}p \\ e_{1}, \ldots, e_{p}\end{array}\right)$ (with $e_{1}, \ldots, e_{p}<p$ ) is zero in $\mathbb{k}=R / \hbar R$, i.e. $\left(\begin{array}{c}p \\ e_{1}, \ldots, e_{p}\end{array}\right) \in \hbar R$ : then $\sum_{\substack{e_{1}, \ldots, e_{p}<p \\ e_{1}+\cdots e_{p}=p}}\left(\begin{array}{c}p \\ e_{1}, \ldots, e_{p}\end{array}\right) \sum_{\cup_{k} \Lambda_{k}=\{1, \ldots, n\}} \prod_{k=1}^{p} \delta_{\Lambda_{k}}(\eta)^{e_{k}} \in \hbar^{n+1} H^{\otimes n}$. Finally, $J_{H^{\prime}} \subseteq \hbar J_{H}$ implies $\hbar J_{H^{\prime}}^{\otimes n} \subseteq \hbar^{n+1} H^{\otimes n}$. Thus $\delta_{n}\left(\eta^{p}\right) \in \hbar^{n+1} H^{\otimes n}$ for all $n$, so $\eta \in \hbar H^{\prime}$. 


\section{$\S 4$ Drinfeld's functors on quantum groups}

Lemma 4.1. Let $F_{\hbar} \in \mathcal{Q F \mathcal { A }}$, with $\left.F_{\hbar}\right|_{\hbar=0}$ reduced. Let $I:=I_{F_{\hbar}}$, let $\widehat{F_{\hbar}}$ be the $I$-adic completion of $F_{\hbar}, \widehat{R}$ the $\hbar$-adic completion of $R$, and $\widehat{I^{n}}$ the $I$-adic closure of $I^{n}$ in $\widehat{F_{\hbar}}$, for all $n \in \mathbb{N}$.

(a) $\widehat{F_{\hbar}}$ is isomorphic as an $\widehat{R}$-module to a formal power series algebra, say $\widehat{R}\left[\left[\left\{Y_{b}\right\}_{b \in \mathcal{S}}\right]\right]$.

(b) Use a section $\nu: \mathbb{k} \longleftrightarrow R$ of the quotient map $R \longrightarrow R / \hbar R=: \mathbb{k}$ to identify (set-theoretically) $\widehat{F_{\hbar}} \cong \widehat{R}\left[\left[\left\{Y_{b}\right\}_{b \in \mathcal{S}}\right]\right]$ with $\nu(\mathbb{k})\left[\left[\left\{Y_{0}\right\} \cup\left\{Y_{b}\right\}_{b \in \mathcal{S}}\right]\right]$, where $\hbar \cong Y_{0}$. Then $\widehat{(\widehat{I})^{n}}$ and $\widehat{I^{n}}$ coincide with the set of all formal series of (least) degree $n$ in the $Y_{i}$ 's ( $i \in\{0\} \cup \mathcal{S}$ ), for all $n \in \mathbb{N}$.

(c) Let $\mu: F_{\hbar} \longrightarrow \widehat{F_{\hbar}}$ be the natural map. Then $\mu\left(F_{\hbar}\right) \bigcap \widehat{I^{n}}=\mu\left(I^{n}\right)$ for all $n \in \mathbb{N}$.

Proof. Let $\left.F[G] \equiv F_{\hbar}\right|_{\hbar=0}:=F_{\hbar} / \hbar F_{\hbar}$, and let $\widehat{F[G]}=F[[G]]$ be the $\mathfrak{m}$-adic completion of $F[G]$, with $\mathfrak{m}:=\mathfrak{m}_{e}=\operatorname{Ker}\left(\epsilon_{F[G]}\right) \quad($ see $\S 2.1)$. Then $I=\pi^{-1}(\mathfrak{m})$, where $\pi$ is the map $\pi: F_{\hbar} \longrightarrow F_{\hbar} / \hbar F_{\hbar}=$ $F[G]$, and so $\pi$ induces a continuous (specialization) epimorphism $\widehat{\pi}: \widehat{F_{\hbar}} \longrightarrow F[[G]]$.

By construction $\widehat{F_{\hbar}}$ is a topological $\widehat{R}$-module with $\left(\widehat{F_{\hbar}}\right)_{\infty}=\{0\}$. Set also $J:=\operatorname{Ker}\left(\epsilon_{F_{\hbar}}\right)$.

(a) Let $\left\{y_{b}\right\}_{b \in \mathcal{S}}$ be a $\mathbb{k}$-basis of $\mathfrak{m} / \mathfrak{m}^{2}=: \mathfrak{g}^{\times}$; then $F[[G]] \cong \mathbb{k}\left[\left[\left\{Y_{b}\right\}_{b \in \mathcal{S}}\right]\right]$ since $F[G]$ is reduced. For each $b \in \mathcal{S}$, pick a $j_{b} \in \pi^{-1}\left(y_{b}\right) \bigcap J$ and fix a section $\nu: \mathbb{k} \longleftrightarrow R$ as in (b). Now define a continuous morphism of $\widehat{R}$-modules $\Psi: \widehat{R}\left[\left[\left\{Y_{b}\right\}_{b \in \mathcal{S}}\right]\right] \longrightarrow \widehat{F_{\hbar}}$ mapping $Y \underline{e}:=\prod_{b \in \mathcal{S}} Y_{b}^{\underline{e}(b)}$ to $j \underline{e}:=\prod_{b \in \mathcal{S}} j_{b}^{\underline{e}(b)}$ for all $\underline{e} \in \mathbb{N}_{f}^{\mathcal{S}}:=\left\{\sigma \in \mathbb{N}^{\mathcal{S}} \mid \sigma(b)=0\right.$ for almost all $\left.b \in \mathcal{S}\right\}$ (hereafter, monomials like these are ordered w.r.t. any fixed order of the set $\mathcal{S}$ ). Using $\nu$, we can set-theoretically identify $\widehat{R} \cong \nu(\mathbb{k})\left[\left[Y_{0}\right]\right]$ (with $\left.\hbar \cong Y_{0}\right)$, whence a bijection $\nu(\mathbb{k})\left[\left[Y_{0} \cup\left\{Y_{b}\right\}_{b \in \mathcal{S}}\right]\right] \cong \widehat{R}\left[\left[\left\{Y_{b}\right\}_{b \in \mathcal{S}}\right]\right]$ arises.

Is is easy to check that $\Psi$ is surjective. To show that it is injective too, look at graded rings, namely $G_{\hbar}\left(\widehat{F_{\hbar}}\right):=\oplus_{n=0}^{+\infty}\left(\hbar^{n} \widehat{F_{\hbar}} / \hbar^{n+1} \widehat{F_{\hbar}}\right) \cong \mathbb{k}[\bar{\hbar}] \otimes_{\mathbb{k}}\left(\widehat{F_{\hbar}} / \hbar \widehat{F_{\hbar}}\right) \cong(F[[G]])\left[Y_{0}\right]$. In addition, there is an epimorphism of $\widehat{R}$-modules $\Psi: \widehat{R} \otimes_{\mathbb{k}} F[[G]] \longrightarrow \widehat{F_{\hbar}}$ which induces an isomorphism of graded $\mathbb{k}$-algebras $G_{Y_{0}}\left(\widehat{R} \otimes_{\mathbb{k}} F[[G]]\right) \cong G_{\hbar}\left(\widehat{F_{\hbar}}\right)$ : then (cf. [Bo], Ch. III, §2.8) $\Psi$ is an isomorphism too.

(b) Since $\widehat{I}=\widehat{\pi}^{-1}\left(\operatorname{Ker}\left(\epsilon_{F[[G]]}\right)\right)=\operatorname{Ker}\left(\epsilon_{\widehat{F_{\hbar}}}\right)+\hbar \widehat{F_{\hbar}}$, each element of $\widehat{I}$ is expressed, via $\Psi$, by a series of degree at least 1 ; moreover, for all $b, d \in \mathcal{S}$ we have $j_{b} j_{d}-j_{d} j_{b}=\hbar j_{+}$for some $j_{+} \in \operatorname{Ker}\left(\epsilon_{\widehat{F_{\hbar}}}\right)$. Therefore when multiplying $n$ factors from $\widehat{I}$ expressed by $n$ series of positive degree, we can reorder the unordered monomials in the $y_{b}$ 's occurring in the multiplication process and eventually get a formal series — with ordered monomials — of degree at least $n$.

(c) The analysis above shows that the natural map $\mu: F_{\hbar} \longrightarrow \widehat{F_{\hbar}}$ induces $\mathbb{k}$-module isomorphisms $(\widehat{I})^{n} /(\widehat{I})^{n+1} \cong \widehat{(\widehat{I})^{n}} / \widehat{(\widehat{I})^{n+1}} \cong \widehat{I^{n}} / \widehat{I^{n+1}}(n \in \mathbb{N})$, so $G_{I}\left(F_{\hbar}\right)=G_{\widehat{I}}\left(\widehat{F_{\hbar}}\right)$, these being the graded algebras associated to the $I$-adic and the $\widehat{I}$-adic filtration. Moreover, the given description of the $\widehat{I^{n}}$ 's implies $G_{\widehat{I}}\left(\widehat{F_{\hbar}}\right):=\bigoplus_{n=0}^{+\infty} \widehat{I^{n}} / \widehat{I^{n+1}} \cong \mathbb{k}\left[Y_{0},\left\{Y_{b}\right\}_{b \in \mathcal{S}}\right]$ as $\mathbb{k}$-modules, and the same for $G_{I}\left(F_{\hbar}\right)$. It follows that $F_{\hbar} / I^{n} \cong \widehat{F_{\hbar}} / \widehat{I^{n}}$, whence $\mu\left(I^{n}\right)=\widehat{I^{n}} \cap \mu\left(F_{\hbar}\right)$, as claimed.

Lemma 4.2. Let $F_{\hbar} \in \mathcal{Q F \mathcal { A }}$, and assume that $\left.F_{\hbar}\right|_{\hbar=0}$ is reduced. Then:

(a) if $\varphi \in F_{\hbar}$ and $\hbar^{s} \varphi \in I_{F_{\hbar}}^{n} \quad(s, n \in \mathbb{N})$, then $\varphi \in I_{F_{\hbar}}^{n-s}$;

(b) if $y \in I_{F_{\hbar}} \backslash I_{F_{\hbar}}^{2}$, then $\hbar^{-1} y \notin \hbar F_{\hbar}^{\vee}$;

(c) $\left(F_{\hbar}^{\vee}\right)_{\infty}=\left(F_{\hbar}\right)_{\infty}\left(=I_{F_{\hbar}}^{\infty}\right)$.

Proof. (a) Set $I:=I_{F_{\hbar}}$. Consider $I^{\infty}:=\bigcap_{n=0}^{+\infty} I^{n}$ and the quotient Hopf algebra $\bar{F}_{\hbar}:=F_{\hbar} / I^{\infty}$ : then $\bar{I}:=I_{\bar{F}_{\hbar}}=I / I^{\infty}$. By Lemma 3.1(a), $\bar{F}_{\hbar}$ is again a QFA, with $\left.\bar{F}_{\hbar}\right|_{\hbar=0}=\left.F_{\hbar}\right|_{\hbar=0}$ and 
$\bar{I}^{\infty}:=I_{\bar{F}_{\hbar}}{ }^{\infty}=\{0\}$. Now, $\phi \in I^{\ell} \Longleftrightarrow \bar{\phi} \in \bar{I}^{\ell}$ for all $\phi \in F_{\hbar}, \ell \in \mathbb{N}$, with $\bar{\phi}:=\phi+I^{\infty} \in \bar{F}_{\hbar}$. So it is enough to prove the claim for $\bar{F}_{\hbar}$, hence we can assume $I^{\infty}=\{0\}$; then the natural map from $F_{\hbar}$ to its $I$-adic completion $\widehat{F_{\hbar}}$ is injective. By the proof of Lemma 4.1 one has $\widehat{I^{\ell}} \cap F_{\hbar}=I^{\ell}$, for all $\ell$ : then, thanks to Lemma $4.1(b), \varphi \in F_{\hbar}$ and $\hbar^{s} \varphi \in I^{n}$ imply $\varphi \in \widehat{I^{n-s}} \bigcap F_{\hbar}=I^{n-s}$.

(b) Let $y \in I_{F_{\hbar}} \backslash I_{F_{\hbar}}^{2}$. Assume $\hbar^{-1} y=\hbar \eta$ for some $\eta \in F_{\hbar}^{\vee} \backslash\{0\}$. As $F_{\hbar}^{\vee}:=\bigcup_{N \geq 0} \hbar^{-N} I_{F_{\hbar}}^{n}$ we have $\eta=\hbar^{-N} i_{N}$ for some $N \in \mathbb{N}_{+}, i_{N} \in I_{F_{\hbar}}^{N}$. Then $\hbar^{-1} y=\hbar^{1-N} i_{N}$, so $\hbar^{N-1} y=\hbar i_{N}$ : but the r.-h.-s. belongs to $I_{F_{\hbar}}{ }^{N+1}$, whilst the 1.-h.-s. cannot belong to $I_{F_{\hbar}}{ }^{N+1}$, due to $(a)$ and $y \notin I_{F_{\hbar}}^{2}$.

(c) $F_{\hbar} \subseteq F_{\hbar}^{\vee}$ implies $\left(F_{\hbar}\right)_{\infty} \subseteq\left(F_{\hbar}^{\vee}\right)_{\infty}$. Conversely, by definition $\left(F_{\hbar}\right)_{\infty}$ is a two-sided ideal of $F_{\hbar}$ and $F_{\hbar}^{\vee}$, and $\bar{F}_{\hbar}^{\vee} \equiv\left(F_{\hbar} /\left(F_{\hbar}\right)_{\infty}\right)^{\vee}=F_{\hbar}^{\vee} /\left(F_{\hbar}\right)_{\infty}$, so $\left(F_{\hbar}^{\vee}\right)_{\infty} \bmod \left(F_{\hbar}\right)_{\infty} \subseteq\left(\bar{F}_{\hbar}^{\vee}\right)_{\infty}$ with $\bar{F}_{\hbar}:=F_{\hbar} / I_{F_{\hbar}}^{\infty}=F_{\hbar} /\left(F_{\hbar}\right)_{\infty}$ (a QFA, by Lemma 3.1 $\left.(a)\right)$. So it's enough to show $\left(\bar{F}_{\hbar}^{\vee}\right)_{\infty}^{\infty}=\{0\}$.

Let $\mu: F_{\hbar} \longrightarrow \widehat{F_{\hbar}}$ be as above: it embeds $\bar{F}_{\hbar}$ into $\widehat{F_{\hbar}}$, and gives $\bar{F}_{\hbar}^{\vee} \subseteq{\widehat{F_{\hbar}}}^{\vee}:=\bigcup_{n \geq 0} \hbar^{-n} \widehat{I^{n}}$, so $\left(\bar{F}_{\hbar}^{\vee}\right)_{\infty} \subseteq\left(\widehat{F}_{\hbar}^{\vee}\right)_{\infty}$. By Lemma 4.1, ${\widehat{F_{\hbar}}}^{\vee}$ is contained in the $R$-subalgebra of $F(R) \otimes_{R} \widehat{F_{\hbar}}$ generated by $\left\{\hbar^{-1} j_{b}\right\}_{b \in \mathcal{S}}$, which is polynomial; then $\left({\widehat{F_{\hbar}}}^{\vee}\right)_{\infty}=\{0\}$, so $\left(\bar{F}_{\hbar}^{\vee}\right)_{\infty}=\{0\}$ too.

Proposition 4.3. Let $\operatorname{Char}(\mathbb{k})=0$. Let $F_{\hbar} \in \mathcal{Q F \mathcal { A }}$. Then $\left(F_{\hbar}^{\vee}\right)^{\prime}=F_{\hbar}$.

Proof. Proposition 3.3 gives $F_{\hbar} \subseteq\left(F_{\hbar}^{\vee}\right)^{\prime}$, and we must prove the converse. Let $\bar{F}_{\hbar}:=F_{\hbar} /\left(F_{\hbar}\right)_{\infty}$; then $\left(F_{\hbar}^{\vee}\right)_{\infty}=\left(F_{\hbar}\right)_{\infty}$, by Lemma $4.2(c)$, so $\left(\bar{F}_{\hbar}\right)^{\vee}=F_{\hbar}^{\vee} /\left(F_{\hbar}^{\vee}\right)_{\infty}$, and $\left(\left(\bar{F}_{\hbar}\right)^{\vee}\right)^{\prime}=\left(F_{\hbar}^{\vee}\right)^{\prime} /\left(F_{\hbar}\right)_{\infty}$. Thus, if the claim is true for $\bar{F}_{\hbar}$ then $F_{\hbar} /\left(F_{\hbar}\right)_{\infty}=: \bar{F}_{\hbar}=\left(\left(\bar{F}_{\hbar}\right)^{\vee}\right)^{\prime}=\left(F_{\hbar}^{\vee}\right)^{\prime} /\left(F_{\hbar}\right)_{\infty}$, hence $\left(F_{\hbar} \vee{ }^{\prime}=F_{\hbar}\right.$. So a proof for $\bar{F}_{\hbar}$ is enough, thus we assume $I^{\infty}=\left(F_{\hbar}\right)_{\infty}=\left(F_{\hbar}^{\vee}\right)_{\infty}=\{0\}$, for $I:=I_{F_{\hbar}}$.

Let $x^{\prime} \in\left(F_{\hbar}^{\vee}\right)^{\prime}$; since $\left(F_{\hbar}\right)_{\infty}=\{0\}$ there are $n \in \mathbb{N}$ and $x^{\vee} \in F_{\hbar}^{\vee} \backslash \hbar F_{\hbar}^{\vee}$ such that $x^{\prime}=\hbar^{n} x^{\vee}$. By Theorem 3.4, $F_{\hbar}^{\vee}$ is a QrUEA with $\left.F_{\hbar}^{\vee}\right|_{\hbar=0}=U(\mathfrak{g}), \mathfrak{g}=I^{\vee} /\left(\hbar F_{\hbar}^{\vee} \cap I^{\vee}\right)$, and $I^{\vee}:=\hbar^{-1} I$.

Fix an ordered $\mathbb{k}$-basis $\left\{b_{\lambda}\right\}_{\lambda \in \Lambda}$ of $\mathfrak{g}$, a subset $\left\{x_{\lambda}^{\vee}\right\}_{\lambda \in \Lambda}$ of $I_{F_{\hbar}}^{\vee}$ such that $x_{\lambda}^{\vee} \bmod \hbar F_{\hbar}^{\vee}=b_{\lambda}$ for all $\lambda \in \Lambda$, and set $x_{\lambda}=\hbar x_{\lambda}^{\vee} \in J$, for all $\lambda$. If $d:=\partial(\bar{x})$ is the degree of $\bar{x}$ w.r.t. the standard filtration of $U(\mathfrak{g})$, then (by [EK], Lemma 4.12, or $[\mathrm{KT}], \S 3.8) d:=\partial(\bar{x}) \leq n$. So we can write $\overline{x^{\vee}}$ as a polynomial $P\left(\left\{b_{\lambda}\right\}_{\lambda \in \Lambda}\right)$ of degree $d \leq n$; hence $x^{\vee}=P\left(\left\{x_{\lambda}^{\vee}\right\}_{\lambda \in \Lambda}\right)+\hbar x_{[1]}^{\vee}$ for some $x_{[1]}^{\vee} \in F_{\hbar}^{\vee}$. Now $x^{\prime}=\hbar^{n} P\left(\left\{x_{\lambda}^{\vee}\right\}_{\lambda \in \Lambda}\right)+\hbar^{n+1} x_{[1]}^{\vee}$, with $\hbar^{n} P\left(\left\{x_{\lambda}^{\vee}\right\}_{\lambda \in \Lambda}\right) \in F_{\hbar}$ since $P$ has degree $d \leq n$; as $F_{\hbar} \subseteq\left(F_{\hbar}^{\vee}\right)^{\prime}$ (by Proposition 3.3), $x_{1}^{\prime}:=x^{\prime}-\hbar^{n} P\left(\left\{x_{\lambda}^{\vee}\right\}_{\lambda \in \Lambda}\right) \in\left(F_{\hbar}^{\vee}\right)^{\prime}$ and $x_{1}^{\prime}=\hbar^{n+1} x_{[1]}^{\vee}=\hbar^{n_{1}} x_{1}^{\vee}$ for some $n_{1} \in \mathbb{N}, n_{1}>n$, and $x_{1}^{\vee} \in F_{\hbar}^{\vee} \backslash \hbar F_{\hbar}^{\vee}$. We repeat this construction with $x_{1}^{\prime}$ instead of $x^{\prime}, n_{1}$ instead of $n$, etc.: iterating, we get an increasing sequence $\left\{n_{s}\right\}_{s \in \mathbb{N}}$ and a sequence $\left\{P_{s}\left(\left\{X_{\lambda}\right\}_{\lambda \in \Lambda}\right)\right\}_{s \in \mathbb{N}}$ such that $x^{\prime}=\sum_{s \in \mathbb{N}} \hbar^{n_{s}} P_{s}\left(\left\{x_{\lambda}^{\vee}\right\}_{\lambda \in \Lambda}\right)$ and the degree of $P_{s}\left(\left\{X_{\lambda}\right\}_{\lambda \in \Lambda}\right)$ is at most $n_{s}$. By construction $\hbar^{n_{s}} P_{s}\left(\left\{x_{\lambda}^{\vee}\right\}_{\lambda \in \Lambda}\right) \in I_{F_{\hbar}}^{n_{s}}$ for all $s$, so $\sum_{s \in \mathbb{N}} \hbar^{n_{s}} P_{s}\left(\left\{x_{\lambda}^{\vee}\right\}_{\lambda \in \Lambda}\right) \in \widehat{F_{\hbar}}$ (the $I_{F_{\hbar}}$-adic completion of $\left.F_{\hbar}\right), x^{\prime}=\sum_{s \in \mathbb{N}} \hbar^{n_{s}} P_{s}\left(\left\{x_{\lambda}^{\vee}\right\}_{\lambda \in \Lambda}\right)$ is an identity in $\widehat{F_{\hbar}}$, and $x^{\prime} \in\left(F_{\hbar}^{\vee}\right)^{\prime} \cap \widehat{F_{\hbar}}$. Now consider the specialization map $\pi:\left.F_{\hbar} \longrightarrow F_{\hbar}\right|_{\hbar=0}=F[G]$ and the embedding $\mu: F_{\hbar} \hookrightarrow \widehat{F_{\hbar}}$ : then $\pi$ extends to $\widehat{\pi}: \widehat{F_{\hbar}} \longrightarrow \widehat{F[G]}=F[[G]]$, and $\left.\mu\right|_{\hbar=0}: F[G]=\left.\left.F_{\hbar}\right|_{\hbar=0} \longrightarrow \widehat{F_{\hbar}}\right|_{\hbar=0}=F[[G]]$ is injective too. Since $\operatorname{Ker}(\pi)=\hbar F_{\hbar}$ and $\operatorname{Ker}(\widehat{\pi})=\hbar \widehat{F_{\hbar}}$, this implies $F_{\hbar} \bigcap \hbar \widehat{F_{\hbar}}=\hbar F_{\hbar}$, whence we get $F_{\hbar} \cap \hbar^{\ell} \widehat{F_{\hbar}}=\hbar^{\ell} F_{\hbar}$ for all $\ell \in \mathbb{N}$. Getting back to $x^{\prime} \in\left(F_{\hbar}^{\vee}\right)^{\prime} \cap \widehat{F_{\hbar}}$, we have $x^{\prime}=\hbar^{-n} y$ for some $n \in \mathbb{N}$ and $y \in F_{\hbar}$; therefore $y=\hbar^{n} x^{\prime} \in F_{\hbar} \bigcap \hbar^{n} \widehat{F_{\hbar}}=\hbar^{n} F_{\hbar}$, so eventually $x^{\prime} \in F_{\hbar}$. 
Proposition 4.4. Let $H, K \in \mathcal{H} \mathcal{A}$, and $\langle\rangle:, H \times K \longrightarrow R$ be a Hopf pairing. Then

(a) $H^{\vee} \subseteq\left(K^{\prime}\right)^{\bullet}$ and $K^{\prime} \subseteq\left(H^{\vee}\right)^{\bullet}$ (and viceversa), and the above induces a pairing $H^{\vee} \times K^{\prime} \rightarrow R$.

(b) If the pairing and its specialization at $\hbar=0$ are both perfect, and $K=H^{\bullet}$, then $K^{\prime}=\left(H^{\vee}\right)^{\bullet}$.

Proof. (a) Scalar extension and restriction give a pairing between $H^{\vee}$ and $K^{\prime}$ : we must prove it is $R$-valued. Let $I=I_{H}$. Pick $c_{1}, \ldots, c_{n} \in I, y \in K^{\prime}$ : then $\left\langle\prod_{i=1}^{n} c_{i}, y\right\rangle=\left\langle\otimes_{i=1}^{n} c_{i}, \Delta^{n}(y)\right\rangle=$ $\sum_{\Psi \subseteq\{1, \ldots, n\}}\left\langle\otimes_{i=1}^{n} c_{i}, \delta_{\Psi}(y)\right\rangle=\sum_{\Psi \subseteq\{1, \ldots, n\}}\left\langle\otimes_{i \in \Psi} c_{i}, \delta_{|\Psi|}(y)\right\rangle \cdot \prod_{j \notin \Psi}\left\langle c_{j}, 1\right\rangle \in \sum_{\Psi \subseteq\{1, \ldots, n\}} \hbar^{n-|\Psi|} R$. $\hbar^{|\Psi|} R=\hbar^{n} R$. The outcome is $\left\langle I^{n}, K^{\prime}\right\rangle \subseteq \hbar^{n} R$, whence $\left\langle\hbar^{-n} I^{n}, K^{\prime}\right\rangle \subseteq R$, for all $n \in \mathbb{N}$; then $H^{\vee} \subseteq\left(K^{\prime}\right)^{\bullet}$ and $K^{\prime} \subseteq\left(H^{\vee}\right)^{\bullet}$, hence $H^{\vee} \times K^{\prime} \longrightarrow F(R)$ takes values into $R$, q.e.d.

(b) Let $\psi \in\left(H^{\vee}\right)^{\bullet}$ : then $\left\langle\hbar^{-s} I^{s}, \psi\right\rangle \in R$, so $\left\langle I^{s}, \psi\right\rangle \in \hbar^{s} R$, for all $s$. For $s=0$ we get $\langle H, \psi\rangle \in R$, thus $\psi \in H^{\bullet}=K$, and so $\delta_{n}(\psi) \in K^{\otimes n}$ for all $n$. Now, $H^{\bullet}=K$ implies $\left(H^{\otimes n}\right)^{\bullet}=K^{\otimes n}$ w.r.t. the induced pairing $H^{\otimes n} \times K^{\otimes n} \longrightarrow R$. Moreover, since $\left\langle 1_{H}, J_{K}\right\rangle=0$ and $\delta_{n}(\psi) \in J_{K}^{\otimes n}$, inverting the previous argument we find $\left\langle H^{\otimes n}, \delta_{n}(\psi)\right\rangle=\left\langle I_{H}^{\otimes n}, \delta_{n}(\psi)\right\rangle \subseteq \hbar^{n} R$ (for all $n$ ): thus $\hbar^{-n} \delta_{n}(\psi) \in\left(H^{\otimes n}\right)^{\bullet}=K^{\otimes n}$, i.e $\delta_{n}(\psi) \in \hbar^{n} K^{\otimes n}$ for all $n$, whence $\psi \in K^{\prime}$.

Proposition 4.5. Let $\operatorname{Char}(\mathbb{k})=0$. Let $U_{\hbar} \in \mathcal{Q} r \mathcal{U E \mathcal { A }}$. Then $\left(U_{\hbar}^{\prime}\right)^{\vee}=U_{\hbar}$.

Proof. If the claim holds for $\bar{U}_{\hbar}:=U_{\hbar} /\left(U_{\hbar}\right)_{\infty}$, then $\bar{U}_{\hbar}=\left(\left(\bar{U}_{\hbar}\right)^{\prime}\right)^{\vee}=\left(U_{\hbar}{ }^{\prime}\right)^{\vee} /\left(U_{\hbar}\right)_{\infty}$ by Lemma $3.1(a)$, thus $\left(U_{\hbar}{ }^{\prime}\right)^{\vee}=U_{\hbar}$. Therefore a proof for $\bar{U}_{\hbar}$ is enough, and we may assume $\left(U_{\hbar}\right)_{\infty}=\{0\}$.

Our plan is to mimic the proof of the same result for quantum groups "à la Drinfeld" in [Ga1], Proposition 3.4 (now we drop the hypothesis $\operatorname{dim}(\mathfrak{g})<+\infty$, with $U(\mathfrak{g})=U_{\hbar} / \hbar U_{\hbar}$, by [Ga1], §3.9).

To simplify notation, set $H:=U_{\hbar}$. Let $\widehat{H}$ and $\widehat{R}$ be the $\hbar$-adic completion of $H$ and $R$. Then $\widehat{H}$ is a topological Hopf $\widehat{R}$-algebra, whose coproduct takes values into $\widehat{H} \widehat{\otimes} \widehat{H}:=H \widehat{\otimes} H$, the $\hbar^{-}$ adic completion of $H \otimes H$; clearly $H$ embeds into $\widehat{H}$ as a (topological) Hopf $R$-subalgebra. Set also $\widehat{H}^{\prime}:=\left\{\eta \in \widehat{H} \mid \delta_{n}(\eta) \in \hbar^{n} \widehat{H}^{\widehat{\otimes} n}\right\}$, and $\left(\widehat{H}^{\prime}\right)^{\times}:=\bigcup_{n \geq 0} \hbar^{-n} I_{\widehat{H}^{\prime}}{ }^{n}\left(\subseteq Q(\widehat{R}) \otimes_{\widehat{R}} \widehat{H}\right)$, where $I_{\widehat{H}^{\prime}}:=\operatorname{Ker}\left(\epsilon_{\widehat{H}^{\prime}}\right)+\hbar \cdot \widehat{H}^{\prime}$. Finally, let $\left(\widehat{H}^{\prime}\right)^{\vee}$ be the $\hbar$-adic completion of $\left(\widehat{H}^{\prime}\right)^{\times}$.

Now consider $\widehat{K}:=\widehat{H}^{*} \equiv \operatorname{Hom}_{\widehat{R}}(\widehat{H}, \widehat{R})$ : it is a complete topological Hopf $\widehat{R}$-algebra, w.r.t. the weak topology, in perfect Hopf pairing with $\widehat{H}$. We set $\widehat{K}^{\times}:=\sum_{n \geq 0} \hbar^{-n} J_{\widehat{K}}^{n}\left(\subseteq Q(\widehat{R}) \otimes_{\widehat{R}} \widehat{K}\right)$, where $J_{\widehat{K}}:=\operatorname{Ker}\left(\epsilon_{\widehat{K}}\right)$, we let $\widehat{K}^{\vee}$ be the $\hbar$-adic completion of $\widehat{K}^{\times}$, and we define $\left(\widehat{K}^{\vee}\right)^{\prime}$ in the obvious way. With the same arguments as for Proposition 4.3 , one proves $\left(\widehat{K}^{\vee}\right)^{\prime}=\widehat{K}$. Like in [Ga1], one shows (as for Proposition 4.4) $\widehat{H}^{\prime}=\left(\widehat{K}^{\vee}\right)^{\bullet}$ and $\widehat{K}^{\vee} \subseteq\left(\widehat{H}^{\prime}\right)^{\bullet}$; moreover $\widehat{H}=\widehat{K}^{*}$, hence $\widehat{K}^{\vee}=\left(\widehat{H}^{\prime}\right)^{\bullet}$. Using this and $\left(\widehat{K}^{\vee}\right)^{\prime}=\widehat{K}$ one proves $\left(\widehat{H}^{\prime}\right)^{\vee}=\widehat{H}$ too (see [Ga1] for details).

Definitions imply $\widehat{H} / \hbar^{n} \widehat{H}=H / \hbar^{n} H$, thus $\hbar^{n} \widehat{H} \cap H=\hbar^{n} H$, for all $n \in \mathbb{N}$; similarly $\hbar^{n} \widehat{H}^{\widehat{\otimes} \ell} \bigcap H^{\otimes \ell}=\hbar^{n} H^{\otimes \ell}$, for all $n, \ell \in \mathbb{N}$, whence $\widehat{H}^{\prime} \cap H=H^{\prime}$. The description of $\widehat{H}^{\prime}$ in [Ga1], $§ 3.5$ (which holds for $\operatorname{dim}(\mathfrak{g})=\infty$ too), tells us that $\widehat{H}^{\prime} \cap \hbar^{n} H=I_{\widehat{H}^{\prime}}^{n}$, and also (acting like in the proof of Lemma 4.1), since $I_{\widehat{H}^{\prime}} \cap H^{\prime}=I_{\widehat{H}^{\prime}} \cap H=I_{H^{\prime}}$, that $I_{\widehat{H}^{\prime}}^{n} \cap H=I_{H^{\prime}}^{n}$ for all $n \in \mathbb{N}$.

Finally, take $\eta \in H \backslash \hbar H$. We can show that there is an $\eta^{\prime} \in I_{\widehat{H}^{\prime}}^{\partial(\bar{\eta})}$ (notation of the proof of Lemma 4.2) such that $\eta^{\prime}=\hbar^{\partial(\bar{\eta})} \eta+\eta_{+}^{\prime}$ for some $\eta_{+}^{\prime} \in I_{\widehat{H}^{\prime}}^{\partial(\bar{\eta})+1}$, just like in [Ga1], §3.5. Roughly, we consider any basis of $\left.H\right|_{\hbar=0}=\left.\widehat{H}\right|_{\hbar=0}$ containing $\bar{\eta}$, we look at the dual basis inside $\left.\widehat{K}\right|_{\hbar=0}$ and lift it to a topological basis of $K$, then rescale the latter (dividing out each element by a proper power of $\hbar$ ) to sort a topological basis of $K^{\vee}$ : the dual basis of $\widehat{H}^{\prime}$ will contain an element $\eta^{\prime}$ as required. Then $\hbar^{\partial(\bar{\eta})} \eta=\eta^{\prime}-\eta_{+}^{\prime} \in I_{\widehat{H}^{\prime}}^{\partial(\bar{\eta})} \cap H=I^{\partial(\bar{\eta})}$, by the previous analysis: so $\eta \in \hbar^{-\partial(\bar{\eta})} I^{\partial(\bar{\eta})} \subseteq\left(H^{\prime}\right)^{\vee}$. Then $H \subseteq\left(H^{\prime}\right)^{\vee}$, whereas the reverse inclusion follows from Proposition 3.3. 
Corollary 4.6. Let $\operatorname{Char}(\mathbb{k})=0$. Let $U_{\hbar} \in \mathcal{Q} r \mathcal{U E \mathcal { A }}$. Then $\left(U_{\hbar}^{\prime}\right)_{F}=\left(U_{\hbar}\right)_{F}$.

Proof. Definitions give $H_{F}^{\vee}=H_{F}$ for all $H$, so $\left(U_{\hbar}^{\prime}\right)_{F}=\left(\left(U_{\hbar}^{\prime}\right)^{\vee}\right)_{F}=\left(U_{\hbar}\right)_{F}$ by Proposition 4.5.

Theorem 4.7. Let $F_{\hbar}[G] \in \mathcal{Q F \mathcal { A }}$ (notation of Remark 1.5) such that $\left.F_{\hbar}[G]\right|_{\hbar=0}$ is reduced. Then we have $\left.F_{\hbar}[G]^{\vee}\right|_{\hbar=0}:=F_{\hbar}[G]^{\vee} / \hbar F_{\hbar}[G]^{\vee}=U\left(\mathfrak{g}^{\times}\right)$as co-Poisson Hopf algebras (see $\S 1.1$ ).

Proof. Set for short $F_{\hbar}:=F_{\hbar}[G], F_{0}:=F_{\hbar} / \hbar F_{\hbar}=F[G]$, and $F_{\hbar}^{\vee}:=F_{\hbar}[G]^{\vee}, F_{0}^{\vee}:=F_{\hbar}^{\vee} / \hbar F_{\hbar}^{\vee}$. By Theorem 3.4, $F_{0} \vee=\mathcal{U}(\mathfrak{k})$ for some $\mathfrak{k}$ (since $\mathbb{k}$ is a field!), and we want to improve this result.

Again, $\left(F_{\hbar}\right)_{\infty}=\left(F_{\hbar}^{\vee}\right)_{\infty}$ by Lemma 4.2(c); then $\overline{F_{\hbar}^{\vee}}:=F_{\hbar}^{\vee} /\left(F_{\hbar}^{\vee}\right)_{\infty}=F_{\hbar}^{\vee} /\left(F_{\hbar}\right)_{\infty}=\left(\overline{F_{\hbar}}\right)^{\vee}$ by Lemma $3.1(a)$, and so $\left.F_{\hbar}^{\vee}\right|_{\hbar=0}=\left.\overline{F_{\hbar}^{\vee}}\right|_{\hbar=0}=\left.\left(\overline{F_{\hbar}}\right)^{\vee}\right|_{\hbar=0}$ by Lemma 3.1(b). Thus it is enough to prove the claim for $\overline{F_{\hbar}}$, i.e. we can assume $\left(F_{\hbar}\right)_{\infty}=\{0\}$. Let $I:=I_{F_{\hbar}}$, and let $\widehat{F_{\hbar}}$ be the $I$-adic completion of $F_{\hbar}$; as $I^{\infty}=\left(F_{\hbar}\right)_{\infty}=\{0\}$, the natural map $F_{\hbar} \longrightarrow \widehat{F}_{\hbar}$ is a monomorphism.

Let $J:=\operatorname{Ker}\left(\epsilon_{F_{\hbar}}\right)$ and $J^{\vee}:=\hbar^{-1} J \subset F_{\hbar}^{\vee}$. Let $\left\{y_{b}\right\}_{b \in \mathcal{S}}$ be a $\mathbb{k}$-basis of $J_{0} / J_{0}^{2}$, with $J_{0}:=\operatorname{Ker}\left(\epsilon_{F[G]}\right)$, and lift it to $\left\{j_{b}\right\}_{b \in \mathcal{S}} \subseteq J$. Using notation of Lemma $4.1, I^{n} / I^{n+1} \cong \widehat{I^{n}} / \widehat{I^{n+1}}$ (for all $n$ ); then Lemma 4.1(b) implies that $I^{n} / I^{n+1}$ has $\mathbb{k}$-basis $\left\{\hbar^{e_{0}} j \underline{e} \bmod I^{n+1} \mid e_{0} \in \mathbb{N}, \underline{e} \in\right.$ $\left.\mathbb{N}_{f}^{\mathcal{S}}, e_{0}+|\underline{e}|=n\right\}$ with $|\underline{e}|:=\sum_{b \in \mathcal{S}} \underline{e}(b)$. As $\hbar^{-n} I^{n+1}=\hbar \cdot \hbar^{-(n+1)} I^{n+1} \equiv 0 \bmod \hbar F_{\hbar} \vee$, we argue that $\hbar^{-n} I^{n} \bmod \hbar F_{\hbar}^{\vee}$ is $\mathbb{k}-$ spanned by $\left\{\hbar^{-|\underline{e}|} j \underline{e} \bmod \hbar F_{\hbar}^{\vee}\left|\underline{e} \in \mathbb{N}_{f}^{\mathcal{S}},\right| \underline{e} \mid \leq n\right\}$ : we claim this is a basis of $\hbar^{-n} I^{n} \bmod \hbar F_{\hbar}^{\vee}$. If not, we find a non-trivial linear combination which is zero: multiplying by $\hbar^{n}$ yields $\gamma_{n} \in I^{n} \backslash I^{n+1}$ with $\hbar^{-n} \gamma_{n} \equiv 0 \bmod \hbar F_{\hbar}^{\vee}$; then $\hbar^{-n} \gamma_{n} \in \hbar \cdot \hbar^{-\ell} I^{\ell}$ for some $\ell \in \mathbb{N}$, so $\hbar^{\ell} \gamma_{n}=\hbar^{1+n} I^{\ell} \subseteq I^{1+n+\ell}$ : then Lemma 4.2(a) yields $\gamma_{n} \in I^{n+1}$, a contradiction.

Now let $j_{\beta}^{\vee}:=\hbar^{-1} j_{\beta}$ for all $\beta \in \mathcal{S}$. Since $j_{\mu} j_{\nu}-j_{\nu} j_{\mu} \in \hbar J$, for any $\mu, \nu \in \mathcal{S}$, we have $j_{\mu} j_{\nu}-j_{\nu} j_{\mu}=\hbar \sum_{\beta \in \mathcal{S}} c_{\beta} j_{\beta}+\hbar^{2} \gamma_{1}+\hbar \gamma_{2}$ for some $c_{\beta} \in R, \gamma_{1} \in J$ and $\gamma_{2} \in J^{2}$, whence $\left[j_{\mu}^{\vee}, j_{\nu}^{\vee}\right]:=j_{\mu}^{\vee} j_{\nu}^{\vee}-j_{\nu}^{\vee} \gamma_{\mu}^{\vee} \equiv \sum_{\beta \in \mathcal{S}} c_{\beta} j_{\beta}^{\vee} \bmod J+J^{\vee} J:$ but $J+J^{\vee} J=\hbar\left(J^{\vee}+J^{\vee} J^{\vee}\right) \subseteq \hbar F_{\hbar}^{\vee}$, so $\left[j_{\mu}^{\vee}, j_{\nu}^{\vee}\right] \equiv \sum_{\beta \in \mathcal{S}} c_{\beta} j_{\beta}^{\vee} \bmod \hbar F_{\hbar}^{\vee}$, hence $\mathfrak{h}:=J^{\vee} \bmod \hbar F_{\hbar}^{\vee}$ is a Lie subalgebra of $F_{0}^{\vee}$. But the latter has $\mathbb{k}$-basis $\left\{\left(j^{\vee}\right)^{\underline{e}} \bmod \hbar F_{\hbar}^{\vee} \mid \underline{e} \in \mathbb{N}_{f}^{\mathcal{S}}\right\}$, hence the PBW theorem gives $F_{0}^{\vee}=U(\mathfrak{h})$ as algebras. Also, the proof of Theorem 3.4 gives $\Delta\left(j^{\vee}\right) \equiv j^{\vee} \otimes 1+1 \otimes j^{\vee} \bmod \hbar\left(F_{\hbar}^{\vee}\right)^{\otimes 2}$ for $j^{\vee} \in J^{\vee}$, so $\Delta(\mathrm{j})=\mathrm{j} \otimes 1+1 \otimes \mathrm{j}$ for $\mathrm{j} \in \mathfrak{h}$, whence $F_{0}^{\vee}=U(\mathfrak{h})$ as Hopf algebras too.

Now, the specialization $\pi^{\vee}: F_{\hbar}^{\vee} \longrightarrow F_{0}^{\vee}=U(\mathfrak{h})$ restricts to $\eta: J^{\vee} \longrightarrow \mathfrak{h}:=J^{\vee} \bmod \hbar F_{\hbar}^{\vee}=$ $J^{\vee} / J^{\vee} \cap\left(\hbar F_{\hbar}^{\vee}\right)=J^{\vee} /\left(J+J^{\vee} J_{\hbar}\right)$, for $J^{\vee} \cap\left(\hbar F_{\hbar}^{\vee}\right)=J^{\vee} \cap \hbar^{-1} I_{F_{\hbar}}^{2}=J_{\hbar}+J^{\vee} J_{\hbar}$ by Lemma 4.2(b). Let $\rho: J_{0} \longrightarrow J_{0} / J_{0}^{2}=: \mathfrak{g}^{\times}$be the projection, and $\nu: \mathfrak{g}^{\times} \longleftrightarrow J_{0}$ a section of $\rho$. The specialization $\pi: F_{\hbar} \longrightarrow F_{0}$ restricts to $\pi^{\prime}: J \longrightarrow J /\left(J \cap \hbar F_{\hbar}\right)=J_{\hbar} / \hbar J_{\hbar}=J_{0}$ : we fix a section $\gamma: J_{0} \longleftrightarrow J_{\hbar}$ of $\pi^{\prime}$. Also, multiplication by $\hbar^{-1}$ yields an $R$-module isomorphism $\mu: J \stackrel{\cong}{\longleftrightarrow}$.

The composition $\sigma:=\eta \circ \mu \circ \gamma \circ \nu: \mathfrak{g}^{\times} \longrightarrow \mathfrak{h}$ is a vector space isomorphism, independent of the choice of $\nu$ and $\gamma$ : we show it is also a Lie bialgebra isomorphism. Pull-back via $\sigma$ the Lie bialgebra structure of $\mathfrak{h}$ onto $\mathfrak{g}^{\times}$, and denote it by $\left(\mathfrak{g}^{\times},[,]_{\bullet}, \delta_{\bullet}\right)$; also, denote by $\left(\mathfrak{g}^{\times},[,]_{\times}, \delta_{\times}\right)$ the Lie bialgebra structure dual to that of $\mathfrak{g}$ : we shall prove that these two structures coincide.

First, for all $x_{1}, x_{2} \in \mathfrak{g}^{\times}$we have $\left[x_{1}, x_{2}\right]_{\bullet}=\left[x_{1}, x_{2}\right]_{\times}$. Indeed, let $f_{i}:=\nu\left(x_{i}\right), \varphi_{i}:=\gamma\left(f_{i}\right)$, $\varphi_{i}^{\vee}:=\mu\left(\varphi_{i}\right), y_{i}:=\eta\left(\varphi_{i}^{\vee}\right) \quad(i=1,2)$. Then $\left[x_{1}, x_{2}\right]_{\bullet}:=\sigma^{-1}\left(\left[\sigma\left(x_{1}\right), \sigma\left(x_{2}\right)\right]_{\mathfrak{h}}\right)=\sigma^{-1}\left(\left[y_{1}, y_{2}\right]\right)=$ $\left(\rho \circ \pi^{\prime} \circ \mu^{-1}\right)\left(\left[\varphi_{1}^{\vee}, \varphi_{2}^{\vee}\right]\right)=\left(\rho \circ \pi^{\prime}\right)\left(\hbar^{-1}\left[\varphi_{1}, \varphi_{2}\right]\right)=\rho\left(\left\{f_{1}, f_{2}\right\}\right)=:\left[x_{1}, x_{2}\right]_{\times}$.

The case of Lie cobrackets is similar, using maps $\nu_{\otimes}:=\nu^{\otimes 2}, \gamma_{\otimes}:=\gamma^{\otimes 2}$, etc., and notation $\chi_{\otimes}:=\eta_{\otimes} \circ \mu_{\otimes}=(\eta \circ \mu)^{\otimes 2}$ and $\nabla:=\Delta-\Delta^{\mathrm{op}}$. Now, for all $x \in \mathfrak{g}^{\times}$we have $\delta_{\bullet}(x)=\delta_{\times}(x)$. Indeed, let $f:=\nu(x), \varphi:=\gamma(f), \varphi^{\vee}:=\mu(\varphi), y:=\eta\left(\varphi^{\vee}\right)$. Then $\delta_{\bullet}(x):=\sigma_{\otimes}{ }^{-1}\left(\delta_{\mathfrak{h}}(\sigma(x))\right)=$ 
$\sigma_{\otimes}^{-1}\left(\delta_{\mathfrak{h}}\left(\eta\left(\varphi^{\vee}\right)\right)\right)=\sigma_{\otimes}^{-1}\left(\eta_{\otimes}\left(\hbar^{-1} \nabla\left(\varphi^{\vee}\right)\right)\right)=\sigma_{\otimes}^{-1}\left((\eta \circ \mu \circ \gamma)_{\otimes}(\nabla(f))\right)=\rho_{\otimes}(\nabla(\nu(x)))=\delta_{\times}(x)$, where the last equality holds because $\delta_{\times}(x)$ is characterized in $\mathfrak{g}^{\times} \otimes \mathfrak{g}^{\times}$by $\left\langle u_{1} \otimes u_{2}, \delta_{\times}(x)\right\rangle=$ $\left\langle\left[u_{1}, u_{2}\right], x\right\rangle$ for all $u_{1}, u_{2} \in \mathfrak{g}$, while $\left\langle\left[u_{1}, u_{2}\right], x\right\rangle=\left\langle\left[u_{1}, u_{2}\right], \rho(f)\right\rangle=\left\langle u_{1} \otimes u_{2}, \rho_{\otimes}(\nabla(\nu(x)))\right\rangle$.

Theorem 4.8. Let $\operatorname{Char}(\mathbb{k})=0$. Let $U_{\hbar}(\mathfrak{g}) \in \mathcal{Q} r \mathcal{U E \mathcal { A }}$ (notation of Remark 1.5). Then we have $\left.U_{\hbar}(\mathfrak{g})^{\prime}\right|_{\hbar=0}:=U_{q}(\mathfrak{g})^{\prime} / \hbar U_{\hbar}(\mathfrak{g})^{\prime}=F\left[G^{\star}\right]$ as Poisson Hopf algebras (see $\left.§ 1.1\right)$.

Proof. By Theorem 3.5, $U_{\hbar}(\mathfrak{g})^{\prime}$ is a QFA, with $U_{\hbar}(\mathfrak{g})^{\prime} \stackrel{\hbar \rightarrow 0}{\longrightarrow} F[H]$; we must show $H=G^{\star}$. Theorem 4.7 applied to $U_{\hbar}(\mathfrak{g})^{\prime}$ yields $\left(U_{\hbar}(\mathfrak{g})^{\prime}\right)^{\vee} \stackrel{\hbar \rightarrow 0}{\longrightarrow} U\left(\mathfrak{h}^{\times}\right)$; then $U(\mathfrak{g}) \stackrel{0 \leftarrow \hbar}{\stackrel{0 \leftarrow}{\longleftarrow}} U_{\hbar}(\mathfrak{g})=\left(U_{\hbar}(\mathfrak{g})^{\prime}\right)^{\vee} \stackrel{\hbar \rightarrow 0}{\longrightarrow} U\left(\mathfrak{h}^{\times}\right)$ by Proposition 4.5 , thus $\mathfrak{h}^{\times}=\mathfrak{g}$ : therefore $\mathfrak{h}:=\left(\mathfrak{h}^{\times}\right)^{\star}=\mathfrak{g}^{\star}$, whence $H=G^{\star}$, q.e.d.

Theorem 4.9. Let Char $(\mathbb{k})=0$. Let $\langle\rangle:, F_{\hbar} \times U_{\hbar} \longrightarrow R$ be a perfect Hopf pairing with $F_{\hbar} \in \mathcal{Q F \mathcal { A }}, U_{\hbar} \in \mathcal{Q} r \mathcal{U E \mathcal { A }}, F_{\hbar}=U_{\hbar}{ }^{\bullet}, U_{\hbar}=F_{\hbar}{ }^{\bullet}$. Then $U_{\hbar}^{\prime}=\left(F_{\hbar}{ }^{\vee}\right)^{\bullet}$ and $F_{\hbar}{ }^{\vee}=\left(U_{\hbar}{ }^{\prime}\right)^{\bullet}$.

Proof. The assumptions imply that the specialized Hopf pairing $\left.F_{\hbar}\right|_{\hbar=0} \times\left. U_{\hbar}\right|_{\hbar=0} \longrightarrow \mathbb{k}$ is perfect as well: then by Proposition 4.4 we have only to prove the inclusion $F_{\hbar}{ }^{\vee} \supseteq\left(U_{\hbar}^{\prime}\right)^{\bullet}$.

Let $\varphi \in\left(U_{\hbar}{ }^{\prime}\right)^{\bullet}$, chosen so that $\left\langle\varphi, U_{\hbar}{ }^{\prime}\right\rangle=R$. Since $\left(U_{\hbar}{ }^{\prime}\right)^{\bullet} \subseteq F(R) \otimes_{R} F_{\hbar}=F(R) \otimes_{R} F_{\hbar}{ }^{\vee}$, there is $c \in R \backslash\{0\}$ such that $\varphi_{+}:=c \varphi \in F_{\hbar}^{\vee} \backslash \hbar F_{\hbar}^{\vee}$ : it follows that $\left\langle\varphi_{+}, U_{\hbar}{ }^{\prime}\right\rangle=c R$. If $F_{\hbar}=F_{\hbar}[G], U_{\hbar}=U_{\hbar}(\mathfrak{g})$, then Theorems $4.7-8$ give $\left.F_{\hbar}^{\vee}\right|_{\hbar=0}=U\left(\mathfrak{g}^{\times}\right)$and $\left.U_{\hbar}^{\prime}\right|_{\hbar=0}=F\left[G^{\star}\right]$. Thus there is $\bar{\eta} \in F\left[G^{\star}\right]$ such that $\left\langle\left.\varphi_{+}\right|_{\hbar=0}, \bar{\eta}\right\rangle=1$, hence there is $\eta \in U_{\hbar}^{\prime}$ (a lift of $\bar{\eta}$ ) such that $\left\langle\varphi_{+}, \eta\right\rangle=1+\hbar \kappa$ for some $\kappa \in R$; but $\left\langle\varphi_{+}, \eta\right\rangle \in c R$, thus $c$ divides $(1+\hbar \kappa)$ in $R$.

As $\varphi_{+} \in F_{\hbar}{ }^{\vee}$ we have $\varphi_{+}=\hbar^{-n} \varphi_{0}$ for some $n \in \mathbb{N}$ and $\varphi_{0} \in I_{F_{\hbar}}^{n}$; therefore $\left\langle\varphi_{0}, U_{\hbar}{ }^{\prime}\right\rangle=c \hbar^{n} R$. On the other hand, as $U_{\hbar}=\left(U_{\hbar}^{\prime}\right)^{\vee}$ (Proposition 4.5) each $y \in U_{\hbar}$ can be written as $y=\hbar^{-\ell} y^{\prime}$ for some $\ell \in \mathbb{N}$ and $y^{\prime} \in U_{\hbar}{ }^{\prime}$; then $\left\langle\varphi_{0}, y\right\rangle=c \hbar^{n-\ell}\left\langle\varphi, y^{\prime}\right\rangle \in R \bigcap c \hbar^{n-\ell} R$ because $\left\langle\varphi_{0}, y\right\rangle \in R$ and $\left\langle\varphi, y^{\prime}\right\rangle \in R$. Now, if $\hbar^{n-\ell}\left\langle\varphi, y^{\prime}\right\rangle \notin R$ then $n-\ell<0$ and so $\hbar$ divides $c$. Since $c$ divides $(1+\hbar \kappa)$ we get an absurd, unless $c$ is invertible in $R$ : then $\varphi=c^{-1} \varphi_{+} \in F_{\hbar}{ }^{\vee}$. Otherwise, we have always $\hbar^{n-\ell}\left\langle\varphi, y^{\prime}\right\rangle \in R$, hence $\left\langle\varphi_{0}, y\right\rangle \in c R$ for all $y \in U_{\hbar}$; thus $c^{-1} \varphi_{0} \in U_{\hbar}^{\bullet}=F_{\hbar}$. Let $\widehat{F_{\hbar}}$ be the $I_{F_{\hbar}}$-adic completion of $F_{\hbar}$ : the natural map $\mu: F_{\hbar} \longrightarrow \widehat{F_{\hbar}}$ has kernel $I_{F_{\hbar}}^{\infty}=\left(F_{\hbar}\right)_{\infty}=\{0\}$ (for $F_{\hbar} \in \mathcal{Q F A}$, and $\left(F_{\hbar}\right)_{\infty}$ is contained in the trivial left radical of the perfect pairing between $F_{\hbar}$ and $U_{\hbar}$; so $F_{\hbar} \subseteq \widehat{F_{\hbar}}$. Now $c^{-1} \varphi_{0} \in F_{\hbar} \subseteq \widehat{F_{\hbar}}$ and $\varphi_{0} \in I_{F_{\hbar}}^{n} \subseteq \widehat{I_{F_{\hbar}}^{n}}$ : then $c^{-1} \varphi_{0} \in \widehat{I_{F_{\hbar}}^{n}}$ by Lemma 4.1 $(a)-$ (b), hence $c^{-1} \varphi_{0} \in \widehat{I_{F_{\hbar}}^{n}} \cap F_{\hbar}=I_{F_{\hbar}}^{n}$, by Lemma 4.1(c). Thus $\varphi=c^{-1} \hbar^{-n} \varphi_{0} \in h^{-n} I_{F_{\hbar}}^{n} \subseteq F_{\hbar}^{\vee}$.

At last, we can gather our partial results to prove the main result, i.e. Theorem 2.2:

Proof of Theorem 2.2. Claim (a) is proved in $\$ \S 3.3-5$, (b) follows from Propositions 4.3 and 4.5, (c) holds by Theorems 4.7-8, whereas Theorem 4.9 proves (d). Finally, assume $\operatorname{Char}(\mathbb{k})=0$ and consider $\mathbb{H} \in \mathcal{H} \mathcal{A}_{F}$. If $H_{(f)} \in \mathcal{Q \mathcal { F }} \mathcal{A}$ (for some prime $\hbar \in R \backslash\{0\}$ ) is an $R$-integer form of $\mathbb{H}$, then $H_{(f)}^{\vee}$ is an integer form too (by the very definitions) and a QrUEA (at $\hbar$ ), by Proposition 3.3. Conversely, if $H_{(u)} \in \mathcal{Q} r \mathcal{U E \mathcal { A }}$ (for some prime $\hbar \in R \backslash\{0\}$ ) is an $R$-integer form of $\mathbb{H}$, then $H_{(u)}^{\prime}$ is an integer form too (by Corollary 4.6) and a QFA (again at $\hbar$ ), by Proposition 3.5. This proves (e).

\subsection{Final remarks:}

(a) The Global Quantum Duality Principle as a "Galois correspondence" theorem. Theorem 2.2 says that Drinfeld's functors set mutually inverse Galois-like correspondences from $\mathcal{H} \mathcal{A}$ to itself. When $(\hbar)$ is maximal, the subcategories of quantum groups then are those of fixed objects for the 
composition of these correspondences. Namely, the composed operator $\left(()^{\vee}\right)^{\prime}=()^{\prime} \circ()^{\vee}$ plays the role of a "closure operator", and $\left(()^{\prime}\right)^{\vee}=()^{\vee} \circ()^{\prime}$ plays the role of a "taking-the-interior operator": then QFAs may be thought of as "closed objects" and QrUEAs as "open objects" in $\mathcal{H} \mathcal{A}$.

(b) Duality between Drinfeld's functors. For $n \in \mathbb{N}$ let $\mu_{n}: J_{H}^{\otimes n} \longleftrightarrow H^{\otimes n} \stackrel{m^{n}}{\longrightarrow} H$ be the composition of the embedding of $J_{H}^{\otimes n}$ into $H^{\otimes n}$ with the $n$-fold multiplication (in $H$ ): then $\mu_{n}$ is the "Hopf dual" to $\delta_{n}$. As $H^{\vee}:=\sum_{n \in \mathbb{N}} \mu_{n}\left(\hbar^{-n} J_{H}^{\otimes n}\right)$ and $H^{\prime}:=\bigcap_{n \in \mathbb{N}} \delta_{n}{ }^{-1}\left(\hbar^{+n} J_{H}^{\otimes n}\right)$, the two functors are built up as "dual" to each other (cf. also part (d) of Theorem 2.2).

(c) Ambivalence QrUEA $\leftrightarrow Q F A$ in $\mathcal{H A}_{F}$. Part (e) of Theorem 2.2 means that some Hopf algebras over $F(R)$ might be thought of both as "quantum function algebras" and as "quantum enveloping algebras": examples are $U_{F}$ and $F_{F}$ for $U \in \mathcal{Q} r \mathcal{U E \mathcal { A }}$ and $F \in \mathcal{Q F} \mathcal{A}$.

(d) Drinfeld's functors for algebras, coalgebras and bialgebras. The definition of either of Drinfeld functors requires only half of the notion of Hopf algebra. In fact, one can define ()$^{\vee}$ for all "augmented algebras" (i.e., roughly speaking, "algebras with a counit") and ( )' for all "coaugmented coalgebras" (roughly, "coalgebras with a unit"), and in particular for bialgebras: this yields nice functors, and neat results extending the global quantum duality principle (cf. [Ga2]).

(e) Generalizations. A good deal of the results about Drinfeld's functors can be extended to the case when $R / \hbar R$ is not a field, i.e. the (prime) ideal $(\hbar)$ is not maximal. Similar considerations hold for remark $(d)$ too.

\section{REFERENCES}

[Bo] N. Bourbaki, Commutative Algebra, Springer \& Verlag, New York-Heidelberg-Berlin-Tokyo, 1989.

[CP] V. Chari, A. Pressley, A guide to Quantum Groups, Cambridge University Press, Cambridge, 1994.

[Dr] V. G. Drinfeld, Quantum groups, Proc. Intern. Cong. of Math. (Berkeley, 1986), 1987, pp. 798-820.

[EH] B. Enriquez, G. Halbout, An $\hbar$-adic valuation property of universal $R$-matrices, J. Algebra 261 (2003), 434-447.

[EK] P. Etingof, D. Kazhdan, Quantization of Lie bialgebras, I, Selecta Math. (N.S.) 2 (1996), 1-41.

[Ga1] F. Gavarini, The quantum duality principle, Annales de l'Institut Fourier 52 (2002), 809-834.

[Ga2] _ The global quantum duality principle: theory, examples, and applications, electronic preprint http://arxiv.org/abs/math.QA/0303019 (2003).

[Ga3] - The Crystal Duality Principle: from Hopf Algebras to Geometrical Symmetries, Journal of Algebra 285 (2005), 399-437.

[Ga4] - Poisson geometrical symmetries associated to non-commutative formal diffeomorphisms, Communications in Mathematical Physics 253 (2005), 121-155.

[KT] C. Kassel, V. Turaev, Biquantization of Lie bialgebras, Pac. Jour. Math. 195 (2000), $297-369$.

[Mo] S. Montgomery, Hopf Algebras and Their Actions on Rings, CBMS Regional Conference Series in Mathematics 82, American Mathematical Society, Providence, RI, 1993.

FABIO GAVARINI

Università degli Studi di Roma "Tor Vergata"

Dipartimento di Matematica

Via della Ricerca Scientifica 1, I-00133 Roma, ITALY

E-MAIL:GAVARINI@MAT.UNIROMA2.IT 